\title{
Las palmeras de América del Sur: diversidad, distribución e historia evolutiva
}

\author{
The palms of South America: diversity, distribution and evolutionary history
}

\author{
Jean-Christophe Pintaud', Gloria Galeano ${ }^{2}$, Henrik Balslev ${ }^{3}$, Rodrigo Bernal ${ }^{4}$, Finn \\ Borchsenius $^{5}$, Evandro Ferreira ${ }^{6}$, Jean-Jacques de Granville ${ }^{7}$, Kember Mejía ${ }^{8}$, Betty \\ Millán ${ }^{9}$, Mónica Moraes ${ }^{10}$, Larry Noblick ${ }^{11}$, Fred W. Stauffer ${ }^{12}$ y Francis Kahn ${ }^{13}$
}

Trabajo presentado al Simposio Internacional "LAS PALMERAS EN EL MARCO DE LA INVESTIGACIÓN PARA EL DERCO DE IA N AMERICA DEL SUR", 07 al 09 de Noviembre 2007 Museo de Historia Natural, UniMuseo de Historia Natural, versidad Nacional Mayor de San Marcos, Lima, Perú.

Publicado online: 29/11/2008
1 IRD, UMR DIA-PC/DYNADIV, Laboratoire Genetrop, 911 Avenue Agropolis, BP 64501, 34394 Montpellier Cedex 5, France. Email: jeanchristophe.pintaud@ird.fr

2 Instituto de Ciencias Naturales, Universidad Nacional de Colombia, Apartado 7495, Bogotá, Colombia. Email: gagaleanog@gmail.com 3 Department of Biology, Aarhus Univeristy, Ny Munkegade building 1540, 8000 Aarhus C., Denmark. Email: henrik.balslev@biology.au.dk 4 Instituto de Ciencias Naturales, Universidad Nacional de Colombia, Apartado 7495, Bogotá, Colombia.

5 Department of Biology, Aarhus Univeristy, Ny Munkegade building 1540, 8000 Aarhus C., Denmark

6 Instituto Nacional de Pesquisas da Amazônia (INPA), Núcleo de Pesquisas do Acre, BR 364, Km4, Parque Zoobotânico da Universidade Federal do Acre, CEP 69.915-900, Rio Branco, Brasil.

7 Herbarium CAY, Institut de Recherche pour le Développement (IRD), B.P. 165, 97323 Cayenne Cedex, France.

8 Instituto de Investigaciones de la Amazonía Peruana (IIAP), Av. José A. Quiñones km. 2,5, A.P. 784, Iquitos, Perú.

9 Museo de Historia Natural, Universidad Nacional Mayor de San Marcos, Lima - Perú. Avda. Arenales 1256 Jesús María, Lima 14, Perú.

10 Herbario Nacional de Bolivia, Instituto de Ecología, Universidad Mayor de San Andrés, Casilla 10077 - Correo Central, La Paz, Bolivia.

11 Montgomery Botanical Center, 11901 Old Cutler Road, Miami, Florida 33156, USA.

12 Conservatoire et Jardin Botanique de Genève, CP 60, CH 1292, Chambésy-GE, Suisse.

13 IRD, UMR-DIAPC, Casilla 18-1209, Lima, Perú.

\section{Resumen}

Este artículo presenta un inventario de la flora de palmeras autóctonas de Suramérica, conformada por 457 especies y 50 géneros. Se analiza la distribución de este grupo vegetal en siete entidades fitogeográficas y se discuten los principales factores que influyen sobre la evolución de las palmeras en América del Sur.

Palabras clave: América del Sur, palmeras (Arecaceae), fitogeografía, endemismo, flora.

\section{Abstract}

This article presents an inventory of South American palms including 457 species and 50 genera. The distribution of palms within seven phytogeographical entities is analyzed. Factors which influence the evolution of palms in South America are discussed.

Key words: South America, palms (Arecaceae), phytogeography, endemism, flora.

\section{Introducción}

Dentro del contexto global de la distribución de las palmeras, la cual puede ser caracterizada como termocosmopolita (entre $44^{\circ} \mathrm{N}$ y $44^{\circ} \mathrm{S}$ ), el continente sudamericano aparece como uno de los mayores centros de riqueza y diversidad para esta familia de plantas. Cuatro de las cinco subfamilias de palmeras existen en América del Sur: Calamoideae, Coryphoideae, Ceroxyloideae y Arecoideae. Tres tribus y subtribus son endémicas o subendémicas de América del Sur: Phytelepheae, Leopoldinieae y Mauritiinae (Dransfield et al., 2005), mientras algunos otros grupos Neotropicales presentan su mayor desarrollo en América del Sur (Iriarteae, Euterpeae, Manicarieae, Bactridinae), o no existen en otras partes del hemisferio occidental (Ceroxyleae). Dieciocho géneros son endémicos de América del Sur y algunos otros se destacan por su diversificación a nivel específico: Geonoma (69 spp.), Bactris (61 spp.), Attalea (56 spp.), Astrocaryum (39 spp.), Syagrus (35 spp.). En total, el continente suramericano contiene 459 especies que representan 50 géneros (Tabla 1).

Los patrones de riqueza y diversidad de las palmeras en América del Sur pueden interpretarse en base a factores ecológicos actuales, en particular relacionados con el clima (Bjorholm et al., 2005), así como en relación a la historia del grupo (Bjorholm et al., 2006). Cuando las palmeras aparecieron, durante el Cretácico inferior (Bremer, 2000), América del Sur era todavía parte del super-continente austral Gondwana. Es probable que elementos autóctonos gondwánicos permanezcan en la actualidad en América del Sur (Moore, 1973, Bjorholm et al., 2006). El papel de los antiguos eventos de vicarianza entre continentes ha sido muy enfatizado al momento del descubrimiento de la tectónica de las placas (Raven \& Axelrod, 1972). Sin embargo, el efecto de estos eventos sumamente antiguos sobre la distribución actual de las palmeras parece cada vez menos importante, debido al crecimiento de argumentos a favor de migraciones posteriores a larga distancia, basados en particular en la teoría del reloj molecular (Trénel et al., 2007, Cuenca et al., 2008). La cuestión es particularmente crítica en cuanto a las relaciones entre África y América del Sur. La separación entre los dos continentes tuvo lugar hace 95 millones de ańos con la ruptura del último punto de contacto, entre el noreste de Brasil y África occidental. Sin embargo, los dos continentes permanecieron cercanos a este nivel hasta el fin del Paleoceno (55 MA), y las posibilidades de migraciones a través del Atlántico abierto se mantuvieron para numerosos grupos de organismos, tanto animales como vegetales (Pennington \& Dick, 2004, Poux et al., 2006, Renner, 2004). Algunos grupos de palmeras presentan una distribución trans-Atlántica. En la subfamilia Calamoideae, mayormente Paleotropical, la tribu Lepidocaryeae incluye una subtribu Africana (Ancistrophyllinae), otra suramericana (Mauritiinae) y una compartida entre los dos continentes (Raphiinae). Raphia taedigera es claramente un elemento de origen Africano reciente, y su estado de planta indígena en América es aún debatido 
Tabla 1. Sistemática de las palmeras de América del Sur según el sistema de Dransfield et al. 2005 ( ${ }^{*}$ género endémico; sp. ${ }^{1)}$ número de especies suramericanas).

\begin{tabular}{|c|c|c|c|}
\hline Clasificación supragenérica & Género & $\mathrm{sp} \cdot{ }^{1)}$ & Distribución \\
\hline Arecaceae & & & Termocosmopolita \\
\hline Calamoideae & & & Pantropical \\
\hline Lepidocaryeae & & & Anfi-atlántico \\
\hline Raphiinae & Raphia & 1 & Atlántico (origen africano) \\
\hline \multirow[t]{3}{*}{ Mauritiinae } & Lepidocaryum* & 1 & Amazónico \\
\hline & Mauritia & 2 & Amazónico \\
\hline & Mauritiella* & 3 & Trans-andino \\
\hline Coryphoideae & & & Termocosmopolita \\
\hline Sabaleae & Sabal & 1 & Caribeño \\
\hline \multirow[t]{5}{*}{ Cryosophilae } & Chelyocarpus* & 4 & Amazónico/Chocó \\
\hline & Cryosophila & 2 & Centroamericano-Chocó \\
\hline & Itaya* & 1 & Amazónico (oeste) \\
\hline & Trithrinax* & 3 & Suramericano austral \\
\hline & Cocothrinax & 1 & Caribeño \\
\hline \multirow[t]{2}{*}{ Trachycarpeae } & & & Termocosmopolita \\
\hline & Copernicia & 3 & Caribeño y suramericano \\
\hline \multirow{3}{*}{$\begin{array}{l}\text { Ceroxyloideae } \\
\text { Ceroxyleae }\end{array}$} & & & Pantropical (excepto Africa) \\
\hline & & & Pantropical (excepto Africa) \\
\hline & Ceroxylon* & 11 & Andino \\
\hline \multirow[t]{4}{*}{ Phytelephae } & & & Trans-andino \\
\hline & Ammandra* & 1 & Trans-andino \\
\hline & Aphandra* & 1 & Oeste amazónico \\
\hline & Phytelephas & 5 & Trans-andino \\
\hline \multirow{7}{*}{$\begin{array}{l}\text { Arecoideae } \\
\text { Iriarteae }\end{array}$} & & & Pantropical \\
\hline & & & Centro-suramericano (NW) \\
\hline & Dictyocaryum* & 3 & Andino-guyanés-amazónico \\
\hline & Iriartea & 1 & Centro-suramericano (NW) \\
\hline & Iriartella* & 2 & Amazónico \\
\hline & Socratea & 4 & Neotropical \\
\hline & Wettinia & 20 & Andino-subandino \\
\hline \multirow{3}{*}{ Chamaedoreeae } & & & Neotropical+Mascarenas \\
\hline & Chamaedorea & 13 & Centro y suramericano \\
\hline & Wendlandiella* & 1 & Subandino oriental \\
\hline \multirow[t]{2}{*}{ Reinhardtieae } & & & Centroamericano \\
\hline & Reinhardtia & 3 & Centroamericano \\
\hline Cocoseae & & & Pantropical \\
\hline \multirow[t]{9}{*}{ Attaleinae } & & & Pantropical \\
\hline & Allagoptera* & 5 & Suramericano austral \\
\hline & Attalea & 56 & Neotropical \\
\hline & Butia* & 13 & Suramericano austral \\
\hline & Cocos & 1 & Pantropical, origen incierto \\
\hline & Jubaea* & 1 & Andino austral \\
\hline & Lytocaryum ${ }^{*}$ & 2 & Mata Atlántica \\
\hline & Parajubaea* & 3 & Andino \\
\hline & Syagrus & 35 & Suramericano \\
\hline \multirow[t]{3}{*}{ Elaeidinae } & & & Anfi-atlántico \\
\hline & Elaeis & 2 & Anfi-atlántico \\
\hline & Barcella* & 1 & Río Negro \\
\hline Bactridinae & & & Neotropical \\
\hline & Acrocomia & 2 & Neotropical \\
\hline & Aiphanes & 27 & Andino-subandino \\
\hline & Astrocaryum & 39 & Neotropical \\
\hline & Bactris & 61 & Neotropical \\
\hline & Desmoncus & 6 & Neotropical \\
\hline Roystoneae & & & Caribeño \\
\hline & Roystonea & 1 & Caribeño \\
\hline Manicarieae & & & Centro-suramericano (NW) \\
\hline & Manicaria & 1 & Centro-suramericano (NW) \\
\hline Euterpeae & & & Neotropical \\
\hline & Euterpe & 7 & Neotropical \\
\hline & Hyospathe & 6 & Neotropical \\
\hline & Oenocarpus & 9 & Centro-suramericano (NW) \\
\hline & Prestoea & 10 & Montañoso Neotropical \\
\hline Geonomateae & & & Neotropical \\
\hline & Asterogyne & 5 & Centro-suramericano (NW) \\
\hline & Geonoma & 69 & Neotropical \\
\hline & Pholidostachys & 4 & Centro-suramericano (NW) \\
\hline & Welfia & 1 & Centro-suramericano (NW) \\
\hline & Calyptogyne & 2 & Centroamericano-Chocó \\
\hline Leopoldinieae & & & Río Negro \\
\hline & Leopoldinia* & 3 & Río Negro \\
\hline
\end{tabular}


(Henderson et al., 1995). La presencia de las Mauritiinae en Suramérica es, en cambio, de gran antigüedad, y representa un elemento muy original, siendo el único grupo de Calamoideae con hojas palmadas (Baker et al., 2000 a, b). A excepción del enigmático Cocos nucifera, la tribu Cocoseae (Arecoideae) presenta un patrón similar de distribución transatlántica. La subtribu Elaeidinae es compartida entre África y América. Un género es endémico de América del Sur (Barcella) y el otro, Elaeis, presenta una especie africana (E. guineensis) y una especie americana (E. oleifera). Varios análisis moleculares mostraron una gran cercanía filogenética entre las dos especies (Barcelos et al., 1999; Billotte et al., 2001), lo que sugiere una migración trans-Atlántica reciente, cuyo sentido (de oeste a este o al revés), no está claro todavía. La subtribu Attaleinae es mayormente diversificada en América del Sur, pero hay un género relictual en África del Sur, Jubaeopsis, y dos en Madagascar (Voanioala y Beccariophoenix). Gunn (2004) planteó la hipótesis de un origen suramericano de la tribu Cocoseae y de una colonización temprana de la región Paleotropical via el corredor Antártico, aunque una probable dispersión en el sentido opuesto no puede ser descartada. Finalmente, en la tribu Chamaedoreeae, existe una gran discontinuidad geográfica entre el género Hyophorbe endémico de las islas Mascarenas al este de Madagascar y el resto de la tribu, ampliamente distribuido en el Neotrópico (Cuenca \& Asmussen, 2007). En este caso también, una dispersión a través del Atlántico durante el Eoceno tuvo lugar, pero como en los casos anteriores, el sentido (de oeste a este o en sentido contrario) no está definido (Cuenca et al., 2008).

La flora de palmeras de América del Sur tiene también algunas afinidades trans-Pacíficas. La tribu Ceroxyleae incluye, además del aislado Ravenea en Madagascar, un clado conformado por un género de Australia (Oraniopsis), uno de las islas Juan Fernández (Juania), y uno de los Andes (Ceroxylon). Esta distribución ha sido considerada como un caso de vicarianza gondwánica (Uhl \& Dransfied, 1987). A pesar de las enormes distancias que separan estos géneros, un reciente estudio de filogenia molecular (Trénel et al., 2007), interpreta esta distribución como el resultado de migraciones a larga distancia de la época terciaria.

Las tribus Leopoldinieae y Euterpeae, cuyas relaciones siguen siendo oscuras (Asmussen et al., 2006), podrían tener también afinidades trans-Pacíficas, con las tribus Pelagodoxeae o Areceae (Norup et al., 2006).

Finalmente, la flora de palmeras de América del Sur se enriqueció de elementos provenientes del continente norteamericano, aún cuando no existía la conexión terrestre entre los dos continentes. El género Copernicia, único miembro de la tribu Trachycarpeae en América del Sur, es un ejemplo de estas migraciones tempranas desde el Caribe. La tribu Cryosophileae es otro ejemplo de dispersión antigua entre los dos continentes, pero su sentido (de norte a sur o al revés) no se puede definir con certeza todavía, aunque Bjorholm et al. (2006) sugieren un origen boreo-tropical de todos los Coryphoideae. En la tribu Chamaedoreeae, hubo también migraciones entre los dos continentes durante el Terciaro (Cuenca et al., 2008). El establecimiento de la conexión terrestre a nivel del istmo de Panamá hace tres millones de años facilitó considerablemente la entrada de géneros centroamericanos hacia la costa Pacífica (Reinhardtia, Synechanthus), la costa caribeña (Sabal), los valles interandinos (Cryosophila), los Andes y de ahí hacia la Amazonía
(Chamaedorea pinnatifrons). El arco de las Antillas Menores fue seguramente la vía de migración de Cocothrinax barbadensis y Roystonea oleracea hacia el continente suramericano y de Geonoma undata en el sentido opuesto.

La supervivencia y el desarrollo de todos estos linajes en América del Sur se deben a varios factores. La permanencia del bosque tropical húmedo, a pesar de fluctuaciones tanto durante el Terciario como el Cuaternario (Haffer, 1969; Wilf et al., 2003) aseguró el hábitat de una familia básicamente de bosque tropical húmedo. El levantamiento de los Andes introdujo una dinámica y diversidad ambiental muy favorable a la diversificación de las palmeras. Algunos linajes originarios de los Andes se difundieron hasta la costa Pacífica, América Central y la Amazonía occidental (Aiphanes, Wettinia). La orogénesis andina determinó varios casos de vicarianza de época terciaria entre las laderas occidental y oriental de la cordillera, en particular en las Phytelepheae (Trénel et al., 2007). Sin embargo, los Andes actuaron también como una barrera permeable, permitiendo esporádicas migraciones trans-andinas de taxones tropicales. Montúfar (2007) encontró una leve diferenciación genética entre las poblaciones de Oenocarpus bataua de los lados oriental y occidental de los Andes de Ecuador, lo que sugiere el mantenimiento de un flujo génico a través de los Andes Ecuatorianos hasta una época reciente. La distribución de Bactris coloniata (Henderson, 2000), sugiere una dispersión de esta especie del lado occidental hasta el lado oriental. Tales migraciones trans-andinas son aún más evidentes en el caso de taxones de mayor altitud como Aiphanes, Wettinia, Dictyocaryum y Ceroxylon. La problemática de estas migraciones ha sido revisada por Trénel et al., (2008), con referencia al caso de Ceroxylon echinulatum en Ecuador, cuya estructura genética puede explicarse mediante dos migraciones trans-andinas. Finalmente, el corredor caribeño (Haffer, 1970) podría explicar la presencia de taxones tropicales de ambos lados de los Andes y hasta América Central (Iriartea deltoidea, Socratea exorrhiza) sin necesidad de cruzar la cordillera.

El retiro muy progresivo del bosque tropical húmedo a favor de formaciones más secas desde el Eoceno en la parte sur del continente (Jaramillo et al., 2006), dió el tiempo para el desarrollo de una flora muy particular de palmeras de ambientes abiertos. Se destaca el proceso de miniaturización y diversificación de los taxones graminiformes (en Butia y Syagrus principalmente pero también en Acrocomia, Allagoptera, Attalea y Astrocaryum).

El conocimiento científico de las palmeras suramericanas fue casi inexistente entre el descubrimiento del continente y la segunda mitad del siglo dieciocho. Jacquin $(1763,1781)$ hizo las primeras descripciones de palmeras suramericanas, basadas en sus propias exploraciones de la región caribeña. El botánico Francés J. Aublet estudió la flora de la Guyana Francesa entre 1762 y 1764 y publicó uno de los primeros trabajos extensivos sobre una flora tropical, incluyendo las palmeras (Aublet, 1775). El matemático, médico y botánico español José Celestino Mutis (1732-1808) residió en Colombia desde 1760. Constituyó un herbario de más de 24000 especímenes, pero su Flora de Bogotá o de Nueva Granada, conteniendo más de 6000 ilustraciones, nunca fue publicada. Sin embargo, el contenido de este trabajo sirvió de base para otros botánicos como Linnaeus hijo, quien incluyó la descripción de Attalea butyracea de Mutis en su Supplementum Plantarum (1781), obra en la cual describió también Mauritia flexuosa. El botánico alemán Joseph Gaertner 
(1732-1791) publicó la descripción de Manicaria saccifera en su obra De fructibus et seminibus plantarum (1788-1791), el primer estudio sistemático de carpología. Hipólito Ruiz fue encargado en 1777 por el gobierno español para estudiar la historia natural de Perú y Chile. Acompañado por el español J. Pavón y el francés J. Dombey, hicieron varias excursiones, alcanzando la Amazonía. En sus obras sobre la flora de Perú y Chile, Ruiz y Pavón (1794, 1798) describieron algunas palmeras muy interesantes y de difícil acceso como Geonoma trigona (Gentry, 1986a), así como los géneros Iriartea y Phytelephas.

El siglo diecinueve empezó con la famosa exploración de América del Sur por parte de Alexander von Humboldt y Aimé Bonpland (1799-1804). En base a los hallazgos de esta expedición, el género Ceroxylon fue establecido en un artículo de Candolle (1804) con la información proporcionada por Bonpland, mientras que las otras novedades fueron descritas posteriormente por Kunth en un artículo en conjunto con los dos exploradores (Humboldt et al., 1816). Pierre Poiteau continuó el estudio de las palmeras de Guyana Francesa durante su estadía entre 1819 y 1822 mientras que G.F.W. Meyer, en su flora de Essequibo (Guyana) establece el género Astrocaryum (Meyer, 1818).

Carl von Martius, el pionero innegable en el conocimiento de las palmeras suramericanas, llegó a Brasil en 1817. En su obra Historia Naturalis Palmarum, publicada de 1823 a 1853, Martius describió gran número de especies nuevas que él mismo recolectó entre 1817 y 1821, durante su exploración de la Amazonía en Brasil y Colombia, acompañado por el zoólogo Spix. Martius describió también palmeras colectadas por Alcide Dessalines D'Orbigny a la vuelta de su periplo por Brasil, Argentina, Chile y Bolivia (Martius, 1844). El conocimiento de las palmeras de la Amazonía occidental, de los Andes tropicales y otras partes del noroeste de América del Sur hasta América Central se extendió en la misma época gracias a las colecciones y trabajos de Poeppig (en Historia Naturalis Palmarum), Karsten (1857) y Oersted (1859).

El estudio de las palmeras de la región amazónica siguió con Alfred Russel Wallace (1853), quien publicó su libro Palm trees of the Amazon and their uses. Richard Spruce (1871) dividió la Amazonía en grandes regiones fitogeográficas con base en la distribución de las palmeras. Drude $(1881,1882)$ hizo una síntesis de los conocimientos taxonómicos de entonces en la Flora Brasiliensis, iniciada por Martius. Barbosa Rodrigues (1903) presentó un panorama bien ilustrado de las palmeras de la Amazonía brasileña. El botánico suizo Emil Hassler (18641937) recolectó intensivamente la flora de Paraguay, incluyendo a las palmeras.

Max Burret, el experto de las palmeras del herbario de Berlín, en la primera mitad del siglo veinte, describió numerosas especies suramericanas en varias publicaciones, incluyendo revisiones de algunos géneros. Burret estudió las colecciones de sus compatriotas y otros europeos que recolectaron intensivamente en América del Sur en esta época, quienes enviaban originales o duplicados de sus colecciones al herbario de Berlín, en particular Hopp, Huber, Huebner, Luetzelburg, Pickel, Schultze-Rhonhof, Tessmann, Ule, Weberbauer y también los norteamericanos Killip y Smith. El incendio del herbario de Berlín en 1943 prácticamente puso fin al monumental trabajo de Burret. Además, este evento perjudicó considerablemente toda la investigación posterior sobre las palmeras suramericanas debido a la pérdida de numerosos tipos de las especies de Burret, cuyas descripciones generalmente carecen de ilustraciones.

Dugand (1940) hizo el listado de las palmeras de Colombia y describió también nuevas especies. Steyermark (1951), MacBride (1960) y Wessels Boer (1965) trataron las palmeras en la flora de Venezuela, Perú y Surinam, respectivamente. Henderson (1995) publicó un tratamiento florístico completo de las palmeras amazónicas y Henderson et al. (1995), el Field Guide to the Palms of the Americas. Otros trabajos también contribuyeron en la visión fitogeográfica moderna de las palmeras suramericanas, con las síntesis a nivel regional (Galeano \& Bernal, 1987; Wessels Boer, 1988; Galeano, 1991, 1992; Kahn \& Granville, 1992; Moraes et al., 1995, Granville, 1997; Henderson, 1997; Kahn, 1997; Borchsenius et al., 1998; Stauffer 2000; Moraes, 1999, 2004), las compilaciones de datos de herbarios por región (Henderson \& Balick, 1987; Balslev \& Moraes, 1989; Kahn \& Moussa, 1994; Moussa et al., 1994) y también los análisis de estado de conservación (Bernal, 1989; Borchsenius \& Skov, 1999; Llamozas et al., 2003; Galeano \& Bernal, 2005; Bernal \& Galeano, 2006; Millán, 2006) y los numerosos trabajos de sistemática, descripción de nuevas especies y revalidación de otras, los cuales sirvieron de base para establecer el listado general, sin poder mencionarlos todos.

Trabajos en curso sobre Butia y Syagrus en el Cono Sur (Noblick en prep.), sobre Ceroxylon y Aiphanes en los Andes (Galeano, Borchsenius, en prep.), sobre el género Geonoma (Henderson en prep.) añadirán varias especies al presente listado (Apéndice 1).

El panorama siguiente se refiere al desarrollo potencial de la vegetación natural, es decir lo que sería sin intervención humana. En la actualidad, muchas áreas de bosque o sabanas arbóreas son dominadas por pastizales, mosaicos de cultivos o monocultivos intensivos y hasta urbanizaciones.

\section{La región amazónica}

Géneros: 35; especies: 178 (0-500 m), 195 (0-1000 m); endemismo: $63 \%$.

La región amazónica alberga el ecosistema - bosque tropical húmedo - más estable a escala geológica de América del Sur. A pesar de episodios de transgresión marina de gran amplitud, de cambio de drenaje consecutivo con el levantamiento de los Andes, o de fluctuaciones climáticas, especialmente durante el Pleistoceno, hay evidencias de la permanencia de un bosque húmedo de angiospermas, al menos en algunos sectores, desde la era secundaria (Hooghiemstra, 2002). Debido a esta "macroestabilidad", y a las condiciones favorables al desarrollo de las plantas que ofrece el bosque tropical húmedo, la Amazonía siempre ha sido la cuna de innovación biológica y generadora de biodiversidad (Hooghiemstra 2002). No es una sorpresa entonces encontrar en la Amazonía 70\% de los géneros de palmeras de América del Sur, siete de ellos siendo endémicos de la región, ni tampoco el observar importantes radiaciones a nivel específico, especialmente en los géneros Bactris (41 spp.), Geonoma (29 spp.), Attalea y Astrocaryum (28 spp. cada uno). El alto nivel de endemismo para toda la región (121 spp., 63\% del total) es indicador de su significado como entidad biogeográfica bien individualizada.

La región amazónica baja (< $500 \mathrm{~m}$ de altitud), como es considerada por Henderson (1995), corresponde al área de de- 
sarrollo relativamente continuo del bosque tropical húmedo y a las diversas otras formaciones vegetales asociadas en la cuenca del Río Amazonas y en las Guyanas. Esta región se extiende desde el pie-de-monte oriental de los Andes hasta el Atlántico, y presenta tanto al norte como al sur un patrón complejo y gradual de transición hacia formaciones vegetales más abiertas (sabanas, cerrados, bosque seco). La zona considerada, centrada alrededor de la línea equinoccial, abarca un área considerable de unos 6,5 millones de $\mathrm{km}^{2}$. La familia de las palmeras, por esencia tropical e higrófila, es omnipresente en todos los ecosistemas amazónicos (Kahn \& de Granville, 1992). No obstante, aunque la diversidad local de palmeras puede ser muy elevada en ciertos tipos de bosques, con más de 30 especies diferentes creciendo en un área de media hectárea (Kahn \& Mejía, 1991), la diversidad total de la región es sorprendentemente baja considerando su tamaño y su diversidad a nivel de ecosistemas. Henderson (1995) reporta 151 especies para la región amazónica baja $(0-500 \mathrm{~m})$. En el presente estudio, este número asciende a 178 especies, debido a la descripción posterior de algunas especies, en particular dentro de los géneros Attalea, Bactris y Geonoma (Glassman, 1999; Henderson, 2000; Borchsenius et al., 2001; Henderson et al., 2008) y sobre todo, a un concepto de especies más estrecho. Este valor puede compararse con las 134 especies de palmeras de la flora del Ecuador, es decir 25\% menos especies dentro de un territorio 24 veces más pequeño. Aumentando el área de la Amazonía hasta el contorno de los $1000 \mathrm{~m}$, es decir incluyendo las laderas de los Andes orientales y de las montañas guyanesas, llegamos a 195 especies repartidas en 35 géneros. La delimitación de los 1000 metros corresponde más o menos al límite superior de los géneros tropicales como Astrocaryum, Attalea, Oenocarpus, Mauritia, Mauritiella o Desmoncus, y al límite inferior de los géneros de bosques andinos como Ceroxylon y Dictyocaryum. Por lo tanto, los cálculos florísticos sobre la diversidad amazónica presentados aquí son basados en el área correspondiente al gradiente $0-1000 \mathrm{~m}$.

Algunos patrones biogeográficos son muy evidentes en esta región, a nivel de la distribución de las palmeras. En primer lugar, se destaca la riqueza de la parte occidental de la Amazonía. En esta zona situada al oeste de la longitud $65^{\circ} \mathrm{W}$ (Montúfar \& Pintaud, 2006), se encuentran 130 especies (dos tercios del total), de las cuales 76 son endémicas, incluyendo cinco compartidas con altitudes superiores a $1000 \mathrm{~m}$ de los Andes orientales (Wettinia maynensis y las cuatro especies amazónicas de Aiphanes). Esta riqueza resulta por el aporte del elemento andino y más generalmente montańoso Neotropical, representado en particular por los géneros Wettinia, Dictyocaryum, Aiphanes, Chamaedorea y Prestoea, los cuales alcanzan las llanuras amazónicas adyacentes y cercanas a los Andes, así como algunas especies trans-andinas, las cuales se desarrollan en selva tropical baja en ambas laderas de los Andes (Ammandra decasperma, Bactris coloniata, Geonoma pinnatifrons subsp. pinnatifrons, Pholidostachys synanthera, Syagrus sancona). La Amazonía occidental se distingue también por un endemismo a nivel genérico (Aphandra, Itaya, Wendlandiella) y sobre todo, por un alto nivel de endemismo específico (58\% de las especies reportadas en esta zona). Ciertos endemismos están restringidos a valles interandinos muy aislados de Perú (Attalea cephalotus, A. weberbaueri, Astrocaryum perangustatum, A. carnosum y una especie probablemente nueva de Welfia), o al arco de Iquitos (Astrocaryum macrocalyx, Chelyocarpus repens), lo cual indica la importancia de la orogénesis andina en la especiación. El Arco de Fitzcarrald (Espurt et al., 2007) separa la flora de palmeras noroccidental (ej. Astrocaryum chambira) de la flora suroccidental (ej. Astrocaryum gratum). Además, varias radiaciones de especies (Astrocaryum spp., Geonoma spp., Bactris spp.) han sido probablemente favorecidas por la dinámica ambiental producida por la actividad tectónica (Räsänen et al., 1987; 1990). Ciertos autores enfatizan también el papel de posibles refugios de bosque tropical húmedo al pie de los Andes durante las glaciaciones del Pleistoceno, como factor de conservación de la biodiversidad y de especiación (Haffer, 1969; Hooghiemstra \& van der Hammen, 1998).

Las otras partes de la región amazónica son comparativamente mucho menos diversas, pero se destacan por sus particularidades florísticas. Tal es el caso del escudo guyanés, un basamento geológico muy antiguo (Precámbrico), generando suelos pobres en nutrientes (Steege et al., 2006), cuyos afloramientos son relativamente continuos en la región guyanesa oriental, y a menudo más altos y fragmentados hacia el oeste. Dentro de las especies características de las Guyanas orientales, podemos mencionar Syagrus stratincola, la cual crece sobre los afloramientos graníticos, el sorprendente Asterogyne guianensis, único representante amazónico de este género y varias especies de Astrocaryum (A. paramaca, A. rodriguesii, A. sciophilum). Se destaca también una gran diversidad de especies del género Bactris, incluyendo las especies endémicas Bactris aubletiana, B. pliniana, B. raphidacantha. Bactris es el primer género en número de especies en Guyana Francesa y Suriname, y el segundo, después de Geonoma, en la Amazonía occidental. De las Guyanas occidentales podemos mencionar los endemismos Bactris oligoclada, B. ptariana y B. turbinocarpa. Oenocarpus circumtextus es endémico de un fragmento aislado de la formación geológica guyanesa en la Amazonía occidental. Además de su aislamiento geológico, la región Guyanesa está separada de la cuenca amazónica en su región central y oriental por un corredor seco ocupado por sabanas. Estos factores, más la posible existencia de refugios durante el Pleistoceno (Charles-Dominique et al., 2003) explican la presencia de un total de 16 especies de palmeras endémicas o sub-endémicas. Sin embargo, toda la región guyanesa comparte un gran número de especies con las regiones amazónicas colindantes: la Amazonía oriental (ej. Astrocaryum vulgare, A. paramaca, A. rodriguesii, Attalea dablgreniana, A. spectabilis, G. oldemanii), la Amazonía central (ej. Astrocaryum farinosum, Attalea attaleoides, Bactris acanthocarpoides, B. constanciae, Desmoncus phoenicocarpus, Oenocarpus bacaba, Syagrus inajai) y la Amazonía occidental (Euterpe catinga).

Entre la bipolaridad geológica y florística de las regiones andino-oeste amazónicas y guyanesas, se destaca la región central de la Amazonía. Esta región presenta un endemismo propio (Astrocaryum ferrugineum, A. sociale, Bactris balanophora, B. tefensis, Geonoma aspidiifolia, G. oligoclona, Iriartella setigera, Oenocarpus minor) lo cual puede ser relacionado con particularidades geológicas locales, con la barrera del corredor seco al norte, y posiblemente con la presencia de refugios durante el Pleistoceno (Prance, 1973). Un aspecto muy particular de esta zona es la flora de riberas de aguas negras y sabanas sobre arena blanca (producto de la erosión del escudo guyanés) en la cuenca del Río Negro, incluyendo los géneros endémicos Leopoldinia y Barcella. Dos especies típicas de esos ambientes, Mauritia carana y Mauritiella aculeata son compartidas con formaciones 
similares pero distantes y de origen distinto (formación Pebas) de la Amazonía occidental (Wesselingh et al., 2002). Attalea ferruginea (A. racemosa) y Astrocaryum acaule son también elementos frecuentes de estos ambientes, aunque no restringido a ellos. La flora de bosque húmedo de terra firme, por su parte, presenta una prolongación natural desde la Amazonía occidental hacia la zona central, donde llegan especies como Astrocaryum ulei, Euterpe precatoria, Bactris macroacantha.

La Amazonía oriental se destaca por su flora de palmeras poco diversa y prácticamente desprovista de endemismos (Astrocaryum giganteum), debido a su carácter relativamente homogéneo y reciente de planicie de sedimentación y de inundación alrededor de la boca del río Amazonas, donde se desarrollan extensas poblaciones de Euterpe oleracea y se destaca en el estuario la presencia del elemento transatlántico Raphia. El río Amazonas divide además la región en dos bloques, norte y sur, creando una barrera que impide la migración, en particular, de las especies dispersadas por roedores y otros mamíferos. Henderson (1995) menciona el caso de Oenocarpus bacaba al norte y $O$. distichus al sur, los cuales llegan frente a frente de cada lado del Amazonas. Muchas especies de Astrocaryum presentan este patrón también.

Sin embargo, algunas especies escapan de estas grandes tendencias, como Astrocaryum gynacanthum, ampliamente distribuido en la región pero ausente de un gran sector noroeste, Astrocaryum minus, conocido de dos localidades muy alejadas, una en la Amazonía sur-occidental brasileña y la otra en Guyana Francesa, o Manicaria saccifera, con amplia distribución Neotropical pero esporádica en la Amazonía central y occidental.

Finalmente, quedan menos de 15 especies realmente panamazónicas, entre las cuales podemos mencionar Attalea maripa, Bactris simplicifrons, Desmoncus polyacanthos, Geonoma maxima, Mauritia flexuosa y Mauritiella armata. A esta lista hay que añadir unas 12 especies compartidas entre América Central y la Amazonía, algunas con amplia distribución Neotropical, como Bactris maraja, Desmoncus orthacanthos, Geonoma deversa, Hyospathe elegans, Socratea exorrhiza, y dos especies compartidas con la Mata Atlántica (Bactris acanthocarpa y B. hirta).

A pesar de que la Amazonía esté vista principalmente como un bosque húmedo, hay que recalcar la importancia de las vegetaciones abiertas incluidas. Hemos hablado de ciertas sabanas edáficas (arena blanca hidromórfica, afloramientos graníticos), en las cuales podemos incluir también las sabanas costeras de las Guyanas, las cuales se desarrollan bajo un clima húmedo (2000-2400 mm/año), con una corta estación seca. El suelo es arenoso y generalmente hidromórfico. Bactris campestris es un elemento común de estas sabanas, mientras que Mauritia flexuosa crece en las islas de bosque pantanoso y áreas inundables. Existen formaciones herbáceas de mayor desarollo, con determinismo climático presente o pasado. Al sureste de Venezuela, al sur de las Guyanas y al norte de la Amazonía brasileña (sabanas del Río Branco) existe un conjunto de sabanas de tamaño variable, establecidas sobre el escudo Guayanés, sobre planicies de baja o media altitud, con tepuyes y montańas esparcidas, y corresponden a un corredor relativamente seco dentro de la región amazónica. Este corredor seco forma una barrera entre la región Amazónica en sentido estricto y la región guyanesa, limitando la distribución de varias especies de palmeras como Lepidocaryum tenue (al Sur). Los bosques de galería y de margen de sabanas presentan una flora de palmeras amazónico-guyanesa (ej. Bactris ptariana, Euterpe catinga var. catinga). En límite de estas sabanas, los bosques densos y bajos de las laderas de los tepuyes permanecen casi inexplorados y podrían revelar algunas palmeras interesantes.

Otro aspecto singular de la región amazónica, en su región central y sur, es la presencia de sabanas incluidas con afinidades - tanto fisionómicas como florísticas - con los cerrados. La presencia de estas sabanas aparentemente relictuales ha sido interpretada como la evidencia de un mayor desarrollo de las formaciones abiertas en la Amazonía durante los episodios secos del Pleistoceno (Schnell, 1987). Syagrus comosa y S. petraea entran en el dominio amazónico a favor de tales inclusiones (Henderson, 1995).

En los límites de la región amazónica, tanto al norte, con los Llanos colombianos y venezolanos y con las sabanas interiores y costeras guyanesas, como al sur y al este con los Cerrados, se nota un abrupto decrecimiento de la diversidad de palmeras. Entre las especies típicas de la zona periférica, podemos mencionar Attalea luetzelburgii, A. macrolepis, Bactris campestris, B. major var. major (norte), varias especies con amplia distribución en la periferia sur-oeste, sur y sur-este (Attalea eichleri, A. speciosa, Bactris gasipaes var. chichagui, Oenocarpus distichus) y otras restringidas a las márgenes occidentales (Bactris glaucescens, B. major var. socialis, Geonoma occidentalis) u orientales (Syagrus vermicularis). Sin embargo, algunas de estas especies penetran muy adentro de la Amazonía, como Attalea phalerata y Astrocaryum chonta.

De una manera general, la diversidad de palmeras, al igual que de otras plantas (Steege et al., 2006), sigue estrechamente el gradiente de precipitaciones en la región amazónica y su periferia, siendo las partes más húmedas las más ricas. No obstante, algunos géneros como Attalea (sensu stricto) y Syagrus, siguen una tendencia opuesta, siendo mucho más diversos en las formaciones periféricas secas que dentro de la misma Amazonía.

\section{Las formaciones de la periferia noroeste de la Amazonía y de la costa caribeña}

Géneros: 24; especies: 61 (0-1000 m); endemismo: 18\%.

Esta región está conformada por la planicie caribeña de Colombia (Galeano, 1992), la región correspondiente a la Cordillera de la Costa de Venezuela (Stauffer, 1999) y los Llanos de Colombia y Venezuela, en la cuenca del río Orinoco (región Orinoquía; Galeano, 1992).

La región considerada es amplia y tiene fuertes afinidades con las regiones colindantes que son los Andes, la Amazonía, las islas caribeñas y Centroamérica. Eso explica el bajo endemismo. Los Llanos son incluidos aquí siguiendo Henderson (1995) que no les considera dentro de la Amazonía, al contrario de Galeano (1992). Siendo transicionales, estas sabanas tienen más afinidades amazónicas hacia el sur y más afinidades caribeñas hacia el norte. Copernicia tectorum es seguramente el endemismo más notorio de esta región.

\section{Los bosques húmedos de la región caribeña}

Principalmente localizados en los pie-de-monte de la terminación caribeńa de los Andes y de las cordilleras costeras de Colombia (Sierra de Santa Marta) y de Venezuela (Cordillera de la Costa), presentan un endemismo elevado en los géneros Asterogyne (A. ramosa, A. spicata) y Geonoma (G. paraguanensis, $G$. platybothros, G. simplicifrons, G. spinescens), así como elemen- 
tos caribeños y compartidos entre América central y el norte de América del Sur (Prestoea longepetiolata). La península de Paría en la costa oriental de Venezuela se destaca por su endemismo compartido con la isla vecina de Trinidad (Attalea osmantha, Geonoma vaga, Prestoea pubigera). Otras especies de Attalea del grupo Scheelea del norte de Venezuela y Colombia son todavía muy poco conocidas: $A$. macrocarpa, A. maracaibensis, $A$. wesselsboeri (Glassman, 1999).

\section{Los bosques secos de la región caribeña}

Estos bosques deciduos que se encuentran al norte de Colombia y Venezuela, reciben $800-1000 \mathrm{~mm}$ de lluvia al año y tienen una temporada seca de 5-6 meses. La flora de palmeras de estos bosques es pobre. Galeano (1992) reporta 13 especies en 10 géneros para la región de la planicie del Caribe de Colombia, entre ellos Sabal mauritiiformis, único representante de este género caribeño en América del Sur. Wessels Boer (1988) menciona Copernicia tectorum, el cual puede ser abundante, Acrocomia aculeata y Syagrus stenopetala en estos bosques en Venezuela. Otro elemento típicamente caribeño es Cocothrinax barbadensis en la costa venezolana.

\section{Los Llanos}

Se trata de inmensas sabanas de planicie desarrolladas sobre material sedimentario-aluvial originado a partir de los terrenos elevados circundantes y bajo la influencia de los cambios climáticos del cuaternario. Los Llanos se extienden desde el pie de la Cordillera Oriental de Colombia hasta el medio y bajo Orinoco en Venezuela. Los Llanos son limitados por el bosque amazónico al sur, a nivel del río Guaviare, y por bosques secos espinosos costeros al norte. Stauffer (2007) reporta la presencia de 21 géneros y 31 especies de palmeras en los Llanos venezolanos. Los bosques de galería de la región de los Llanos presentan una flora de palmeras amazónicas (Mauritia flexuosa, Attalea butyracea, A. insignis, A. maripa, Astrocaryum gynacanthum, Bactris acanthocarpa, Desmoncus spp., Euterpe precatoria, Geonoma deversa, Hyospathe elegans, Oenocarpus bataua, O. bacaba, Socratea exorrhiza, entre otras) y caribeña (Roystonea oleracea). Tres especies de Bactris crecen en vegetación abierta: B. campestris, $B$. guineensis y B. major. Existen también formaciones de Attalea, como los 'corobales', extensas poblaciones de Attalea macrolepis en las sabanas del oeste de Bolívar y posiblemente del este de Vichada (Stauffer \& Fariñas, 2006). Las sabanas con alternancia de inundación y sequía son dominadas por Copernicia tectorum en la parte norte de los Llanos.

\section{Las formaciones mesófilas y xéricas de la periferia Sur y Sur-Este de la Amazonía y del Cono Sur}

Géneros: 15; especies: 79; endemismo: 57\% (excluido dominio de la Mata Atlántica, sensu lato, ver Sección 4)

Toda la parte de América del Sur que se encuentra al sur de la Amazonía y al este de los Andes forma un conjunto muy distinto. Eso se debe a la desaparición rápida de los elementos amazónicos ya que géneros ampliamente distribuidos como Desmoncus, Oenocarpus, Mauritia y Mauritiella apenas cruzan los $15^{\circ} \mathrm{S}$ mientras que Euterpe cuenta con una sola especie en esta latitud, Bactris y Geonoma con una especie también cada uno, fuera de la Mata Atlántica. Estas palmeras están reemplazadas por un elemento endémico austral, lo cual incluye cuatro géneros (Allagoptera, Butia, Lytocaryum y Trithrinax), que se desarrolla hasta $35^{\circ}$ S. Butia ocupa la mayor parte de la región, con varias formas biológicas, y las radiaciones adaptativas en este género y también en Attalea, Syagrus, Allagoptera y Astrocaryum, mayormente en vegetación mesófila y seca, explican el número relativamente elevado de especies de esta región considerando su clima algo desfavorable a las palmeras. La extensión y la antigüedad de las formaciones secas en el sur de América del Sur seguramente permitieron el desarrollo de una flora diversificada de palmeras, lo que ocurrió en forma muy limitada en las formaciones secas de pequeña extensión de la costa pacífica (Jubaea chilensis, Aiphanes eggersii) y de los Andes (Parajubaea spp.).

El bosque tropical húmedo de la franja costera atlántica y su prolongación hacia el interior (Mata Atlántica) representa por su lado un fenómeno biogeográfico con una flora de palmeras compuesta por $96 \%$ de especies endémicas de la región sur. Además, el $81 \%$ de las especies, incluyendo radiaciones en Bactris y Geonoma y un género endémico con dos especies (Lytocaryum), son propios del dominio de la Mata Atlántica. Por esta razón, esta entidad está tratada por separado (Sección 4).

En conjunto, las formaciones húmedas, mesófilas y xéricas al sur de la Amazonía y al este de los Andes contienen 123 especies ( $80 \%$ endémicas) perteneciendo a 16 géneros. Este conteo incluye las laderas andinas orientales al sur de la Amazonía hasta los $1000 \mathrm{~m}$ y la totalidad de las montañas del este de Brasil hasta el límite superior de las palmeras (1300-1800 m) en los campos rupestres y bosque atlántico montañoso.

\section{Bosques de transición de la periferia amazónica}

La transición entre la selva amazónica y las formaciones más secas del sur y sur-este se presenta en forma variable. La flora de palmeras amazónicas se extiende dentro del dominio de los Cerrados hasta aproximadamente $15-17^{\circ} \mathrm{S}$, gracias a los bosques de galería, funcionando como refugios edáficos discontinuos, donde permanecen las especies con amplia distribución (Mauritia flexuosa, Mauritiella armata, Oenocarpus distichus, Bactris major, D. orthacanthos, D. polyacanthos), asociadas con especies de la perifieria sur (Attalea princeps, Astrocaryum chonta, A. gratum, A. huaimi, Bactris glaucescens, Geonoma brevispatha) y especies de la Mata Atlántica hacia el Este (Bactris setosa, Euterpe edulis). En el Sur-Este de Madre de Dios (Perú), se observa un límite abrupto entre un bosque tropical con estación seca, caracterizado por especies de palmeras como Attalea moorei, A. phalerata, Astrocaryum gratum, Bactris martiana, Chamaedorea angustisecta, Geonoma occidentalis, Phytelephas macrocarpa, y la pampa de Heath, una sabana con Astrocaryum huaimi, Mauritiella armata y Syagrus sancona, que se prolonga en Bolivia. Los bosques semi-deciduos forman una franja en la periferia sur de la Amazonía (Askew et al., 1970). Estos bosques se caracterizan por un dosel relativamente bajo (15-18 m) y una pluviosidad de 1500 -2000 mm/año, con una estación seca de 6-7 meses. Las palmeras más características de estos bosques son Attalea eichleri, A. speciosa, Acrocomia aculeata, Bactris gasipaes var. chichagui y S. vermicularis (Henderson 1995, Noblick 2004b, Silva \& Clement 2005). En varias partes se observan extensas formaciones dominadas por Attalea speciosa (zona dos cocais en Maranhão, Sampaio 1933), de origen probablemente secundario (Aubreville, 1961; Anderson et al., 1991).

En total la flora de palmeras de bosque al sur de la Amazonía y al oeste de la Mata Atlántica es relativamente pobre con 25 especies. 


\section{Cerrados, catingas y pampas}

El tipo de vegetación tropical abierta más desarrollado al sur de la región amazónica es el Cerrado. Típicamente, el Cerrado se presenta en forma de una sabana con una matriz dominada por cobertura herbácea principalmente compuesta por gramíneas mezclada con especies leñosas de tallo subterráneo, arbustos y árboles esparcidos con tronco tortuoso y corteza gruesa y suberificada. Los Cerrados están asociados a los suelos erosionados y pobres del Escudo Brasileño, en su mayoría por encima de los $500 \mathrm{~m}$ de altitud, bajo un clima con prolongada estación seca y precipitaciones de $700 \_1750 \mathrm{~mm} /$ año. La región de los Cerrados abarca la parte central de Brasil y áreas adyacentes de Paraguay y Bolivia. La flora de palmeras de los Cerrados esta prácticamente desprovista de especies con afinidad amazónica, con pocas de ellas creciendo en la región siendo mayormente limitadas a los bosques de galería y otros lugares húmedos (Attalea phalerata, A. anisitsiana, Bactris glaucescens, Geonoma brevispatha). En cambio, los géneros endémicos de la parte meridional de América del Sur son bien representados (Allagoptera, Butia, Trithrinax), así como Acrocomia, Attalea y Syagrus. Las especies con tallo subterráneo, frecuentemente con crecimiento de tipo 'saxofon' (germinación con geotropismo positivo), o con hábito diminuto y graminiforme, son numerosas en los géneros Acrocomia (A. hassleri), Astrocaryum (Kahn, 2008), Attalea (A. exigua, A. geraensis), Butia (Noblick 2004a, 2006) y Syagrus (S. campylospatha, S. graminifolia, S. lilliputiana, S. petraea, S. werdermannii). Según la reciente síntesis de Moraes (2007), la región del Cerrado en Bolivia comprende 11 especies de vegetación abierta, más 10 especies con afinidad amazónica restringidas a los bosques de galería, ribereños e inundables, y cuatro especies compartidas con los Andes adyacentes (Attalea phalerata, Syagrus cardenasii, S. sancona y S. yungasensis). En Brasil, Rizzini (1963) menciona 20 especies de cerrados herbáceos en seis géneros. Entre Brasil, Bolivia y Paraguay se registran en el presente listado 40 especies en este tipo de vegetación (26 endémicas).

La vegetación xérica de las sierras rocosas del centro-este de Brasil (campo rupestre) incluye especies de Syagrus, varias de aquellas endémicas, creciendo hasta $1200 \_1400 \mathrm{~m}$ de altitud (S. duartei, S. glaucescens, S. harleyi, S. microphylla, S. pleioclada).

Existen también bosques deciduos secos en el dominio de los Cerrados, en particular sobre los afloramientos calcáreos. Attalea brasiliensis es endémico de este tipo de bosque (Glassman, 1999).

En el noreste brasileño, se conoce como caatinga a la vegetación xérica, espinosa, característica de esta región. En la parte central, las precipitaciones son inferiores a $500 \mathrm{~mm} /$ año y el paisaje es particularmente árido (Sertão). Las palmeras del noreste son poco numerosas e incluyen a Syagrus coronata, muy abundante en toda la región, $S$. vagans en catingas arenosas áridas, $S$. microphylla en áreas rocosas, Attalea vitrivir, en bosque de galería y Copernicia prunifera, en zonas con alternancia de inundación en temporada de lluvias y largos períodos de sequía.

$\mathrm{Al}$ sur del continente, las palmeras llegan a latitudes extratropicales $\left(25-35^{\circ} \mathrm{S}\right)$ y alcanzan el dominio de la pampa (sensu Cabrera \& Willink, 1973). La pampa es una vegetación templada (entre $30^{\circ}$ y $40^{\circ} \mathrm{S}$ ) predominantemente herbácea, de transición entre la región Neotropical y la región Neoantártica, donde las palmeras (Butia y Trithrinax) se encuentran en límite austral. La pampa se desarrolla desde el extremo sureste de Brasil hasta el noreste de Argentina y Uruguay. En el suroeste de Rio Grande do Sul (Brasil) y en la parte adyacente de Uruguay, existen las curiosas pampas dominadas por Butia lallemantii.

\section{El Gran Chaco}

Es una región extensa, sub-tropical (entre $18^{\circ}$ y $29^{\circ} \mathrm{S}$ ), limitada al oeste por los Andes, pasando a las pampas al sur y a los cerrados al este. El gran Chaco abarca el sur de Bolivia al este de los Andes, el noroeste de Paraguay, apenas alcanzando las zonas adyacentes de Mato Grosso (Brasil), y une gran parte del norte de Argentina (Chaco meridional). El clima presenta una prolongada estación seca y lluvias de verano, y se caracteriza por una gran amplitud térmica diaria en invierno con más de 45 ${ }^{\circ} \mathrm{C}$ a medio día y menos de $10{ }^{\circ} \mathrm{C}$ en la noche. La vegetación está compuesta principalmente de bosque seco espinoso, con cactáceas y bombacáceas, bosques de quebracho (Schinopsis y Aspidosperma), así como de sabanas aisladas, frecuentemente asociadas con dunas de arena. La palmera típica del bosque seco espinoso es Trithrinax schizophylla, pero se encuentra también Acrocomia aculeata (Moraes, 2007). Sobre los suelos más húmedos, inundados durante la temporada de lluvias, se desarrollan extensas poblaciones de Copernicia alba.

\section{El Pantanal}

Esta región muy característica es localizada al suroeste de Mato Grosso y en la parte adyacente del este de Bolivia. Es una región de lagos, pantanos, sabanas inundadas en temporada de lluvias, y franjas de bosques ribereńos con Bactris y Astrocaryum. Las zonas marginales incluyen formaciones dominadas por $C o-$ pernicia alba (en áreas periódicamente inundadas), Acrocomia aculeata, Attalea eichleri y A. phalerata.

\section{La Mata Atlántica}

Géneros: 10; especies: 35 (sensu stricto, 69\% endémicas) ó 54 (sensu lato, 81\% endémicas).

Se trata del bosque tropical y subtropical húmedo que se desarrolla bajo la influencia de las precipitaciones generadas por la humedad proveniente del Atlántico, desde la costa del noreste brasileño hasta el extremo noreste de Argentina y Uruguay, y hasta el este de Paraguay. Las precipitaciones en la costa pueden sobrepasar los $2000 \mathrm{~mm}$ /año y alcanzan los $4000 \mathrm{~mm}$ o más en las laderas orientales de las cordilleras costeras. Esta zona corresponde a la Mata Atlántica sensu stricto. Hacia el interior, fuera de la influencia directa de la humedad Atlántica, el dominio de la Mata Atlántica sensu lato comprende bosques semi-deciduos y deciduos, en el límite con el dominio de los Cerrados (Ledru et al., 2007). Los bosques con régimen estacional del dominio de la Mata Atlántica incluyen especies como Attalea brejinhoensis, A. compta, A. pindobassu, A. seabrensis, Syagrus cearensis, S. coronata, S. oleracea. El bosque dominado por Araucaria angustifolia es también parte de la Mata Atlántica sensu lato y posee algunas palmeras (Butia eriospatha, Trithrinax brasiliensis), y también las formaciones costeras de restinga (ver Sección 7). La flora de palmeras del bosque tropical húmedo Atlántico incluye elementos típicos de la región sur (Lytocaryum, Allagoptera, Syagrus, Attalea,) pero también un importante contingente claramente vicariante con el bosque amazónico (del cual está separado por las formaciones secas de los Cerrados y Sertão). Pocas especies son compartidas entre ambos bosques (Bactris acanthocarpa y 
$B$. hirta) pero las especies endémicas de los géneros Astrocaryum (A. aculeatissimum) y Bactris (11 spp.) tienen estrechas afinidades con especies amazónicas. El género Geonoma, con ocho especies endémicas, está mayormente compuesto por un grupo de especies afines de $G$. brevispatha (Henderson et al. 1995). El dominio de la Mata Atlántica sensu lato comprende 54 especies, siendo la franja de bosque tropical húmedo costero la más rica (35 especies). Más que el número de especies, es el nivel excepcional de endemismo (81\%), que hace esta zona tan importante en términos de biodiversidad y de conservación.

\section{La costa pacífica, el pie-de-monte andino occidental, la zona del istmo de Panamá y los valles interandinos de Colombia}

Géneros: 31; especies: 105 (0-1000 m); endemismo: 38\%

La región pacífica presenta una mezcla de influencias biogeográficas única en el continente suramericano. Los géneros más diversos en la región son Geonoma (20 spp.), Bactris (12 spp.), Aiphanes (11 spp.), Chamaedorea (8 spp.) y Wettinia (6 spp.). Cinco géneros se encuentran solo en la parte norte de la región: Calyptrogyne (2 spp.), Cryosophila (2 spp.) y Reinhardtia (3 spp.), géneros de filiación centroamericana, cuyas especies del Pacífico demarcan el límite Sur de su distribución, y Elaeis y Raphia, dos géneros compartidos con África. Algunas especies restringidas en Suramérica a la zona del istmo alcanzan el lado atlántico también, como Chamaedorea pygmaea, Reinhardtia spp. y Calyptrogyne costatifrons.

Cuarenta especies son exclusivas de la región del Pacífico; 11 de las 19 especies de Geonoma que crecen en la región son endémicas de ella, al igual que tres de las seis especies de Attalea. Es de destacar también en esta región, la diversificación de dos géneros predominantemente andinos Aiphanes y Wettinia, con cuatro y tres especies exclusivas de la región del Pacífico, respectivamente, así como la presencia de las tres únicas especies de Astrocaryum al oeste de los Andes: A. malybo, A. standleyanum y $A$. triandrum.

El número total de especies de palmeras de la región del Pacífico representa una alta diversidad, teniendo en cuenta el área limitada de la región. De hecho, Bjorholm et al. (2005, 2006), señalaron el área del Chocó como la de mayor expresión de la diversidad en palmeras en América, con 81 especies por cuadrícula de un grado de lado.

Pero esta región, se caracteriza no sólo por su alta riqueza en palmeras, sino también por la gran abundancia en individuos de palmeras por unidad de área, comparada con otras regiones del Neotrópico. Esta abundancia de palmeras le aporta a la región del Pacífico, una fisonomía característica (Gentry, 1986b, 1993; Borchsenius, 1997; Borchsenius et al., 1998; Galeano, 2001).

\section{Los bosques secos y mesófilos de la periferia del desierto costero}

La corriente fría de Humboldt determina la presencia del desierto costero de Perú-Chile, lo que tiene por consecuencia la ausencia total de palmeras de $4^{\circ} \mathrm{S}$ hasta $32^{\circ} \mathrm{S}$, una situación sin equivalente en el resto del mundo. Al sur del desierto existe el aislado Jubaea chilensis, formando poblaciones importantes en el matorral seco del pie de los Andes, entre $32^{\circ} \mathrm{S}$ y $35^{\circ} \mathrm{S}$. Jubaea es el único género de palmeras endémico de la costa Pacífica de América del Sur.
Al norte del desierto se desarrolla la flora tumbesiana, característica de los bosques estacionalmente secos del norte de Perú y Ecuador (Aguirre et al. 2006). El bosque deciduo dominado por malváceas (subfamilia Bombacoideae: Ceiba trichistandra, Cavanillesia platanifolia, Eriotheca ruizii) es la formación más seca donde se encuentran palmeras, recibe $1000 \mathrm{~mm}$ o menos de lluvia al año y soporta $6-7$ meses de estación seca. Este bosque contiene sólo dos palmeras, Aiphanes eggersii y Attalea colenda, ambas endémicas de la costa Pacífica, la segunda restringida a los fondos de valles húmedos. En el norte de Tumbes (Perú), Aiphanes eggersii es un elemento dominante del sotobosque en la zona de transición entre el bosque deciduo y el bosque premontano, alrededor de $600 \mathrm{~m}$ de altitud. El bosque premontano mesófilo contiene una sola especie de palmeras, Chamaedorea linearis (Pintaud \& Millán 2004). En el sur de Ecuador (Loja), el bosque premontano semi-deciduo se desarrolla alrededor de 800—1000 $\mathrm{m}$ de altitud y contiene Bactris gasipaes var. chichagui, Ceroxylon echinulatum y C. parvum. El género Ceroxylon llega a su altitud más baja en este ecosistema, a favor de la alta nebulosidad que compensa los siete meses de estación seca. Un fenómeno similar explica la presencia de Geonoma undata a solo $500 \mathrm{~m}$ de altitud más al norte (Manabí) en las montańas costeras de Chindul. Los bosques mesófilos de baja altitud en Ecuador se extienden a lo largo de la costa, hasta en la parte norte (Esmeraldas), reciben menos de $2000 \mathrm{~mm}$ de lluvia al año, y soportan una estación seca de 4 a 6 meses. Solamente siete especies de palmeras crecen en estos bosques pero representan un conjunto de influencias biogeográfricas muy particular: Aiphanes eggersii es un elemento xérico, única especie de palmeras perteneciendo a la flora endémica tumbesiana; Attalea colenda y Phytelephas aequatorialis son elementos mesófilos endémicos de la parte sur de la región pacífica, y el límite norte de su distribución corresponde a la transición entre el bosque estacionalmente húmedo y el bosque pluvial; Astrocaryum standleyanum es la única especie clinal de la franja pacífica, atravesando todo el gradiente climático desde el bosque perhúmedo hasta la margen del bosque seco; finalmente Bactris coloniata, Bactris gasipaes var. chichagui, y Syagrus sancona son elementos trans-andinos caracterizados por una ecología 'errática', creciendo según los lugares en bosque muy húmedo (Andes orientales del Perú central, Amazonía occidental) o seco (sur de la región pacífica, periferia suroeste de la Amazonía, costa caribeña).

\section{Los bosques del 'Chocó biogeográfico'}

Se trata de los bosques húmedos de las tierras bajas al occidente de los Andes, desde las serranías que forman la frontera entre Colombia y Panamá hasta el noroccidente de Ecuador, en el Sur de Esmeraldas y Pichincha. En su conjunto, esta región, denominada 'Chocó biogeográfico' (Gentry 1982, Forero \& Gentry 1989), constituye la región más lluviosa de América y una de las de mayor pluviosidad en el mundo, con precipitaciones que van hasta $12000 \mathrm{~mm}$ anuales en el centro de la región y disminuyen progresivamente hasta los 3000—4000 mm en sus extremos norte y sur, respectivamente (Eslava 1992, 1993).

El bosque pluvial del Chocó se caracteriza por la ausencia de estación seca, se encuentra principalmente en Colombia, alcanzando el extremo noroeste de Ecuador. El bosque pluvial es la formación más diversa de la costa Pacifica. Se nota la presencia de especies endémicas, con afinidades sea centroamericanas ( $\mathrm{Ca}$ lyptrogyne baudensis, Cryosophila macrocarpa), andinas (Aiphanes 
spp., Wettinia spp.) o amazónicas (Chelyocarpus dianeurus, Mauritiella macroclada).

$\mathrm{Al}$ sur del bosque pluvial se encuentran los bosques estacionalmente húmedos. Este tipo de bosque alto, siempre verde, presenta una gran extensión $\left(32000 \mathrm{~km}^{2}\right)$ en la costa pacífica y en el pie-de-monte andino occidental de Ecuador, y recibe $2000 \_4000 \mathrm{~mm}$ de precipitaciones al año con 2—4 meses de estación seca (Borchsenius et al. 1998). Estos bosques son menos diversos que los precedentes, tanto a nivel específico como genérico, con la ausencia de los cinco géneros restringidos a la parte norte, así como de Ammandra, Asterogyne, Chelyocarpus, Manicaria y Mauritiella, los cuales alcanzan la parte central o hasta el límite sur del bosque pluvial.

Se nota en cambio la presencia de elementos de tendencia mesófila (Attalea colenda, Bactris gasipaes var. chichagui, Phytelephas aequatorialis), más elementos andinos (Chamaedorea linearis, Bactris setulosa), elementos trans-andinos (Iriartea deltoidea, Euterpe precatoria var. longevaginata, Prestoea ensiformis, Oenocarpus bataua, E. mapora, Pholidostachys synanthera, Socratea exorrhiza), una parte de la flora del bosque pluvial del Chocó y de la flora centro-americana (Astrocaryum standleyanum, Bactris coloradonis, Desmoncus cirrhiferus, Geonoma cuneata, Pholidostachys dactyloides, Synechanthus warscewiczianus, Welfia regia, Wettinia aequalis) y unos endemismos locales (Geonoma irena, $G$. tenuissima). Este bosque llega en forma fragmentaria y empobrecida hasta $3^{\circ} 48^{\prime} \mathrm{S}$ en la provincia de El Oro, en proximidad inmediata del bosque seco, a favor de microclimas favorables.

\section{Los valles interandinos de Colombia}

También se incluye en este análisis como parte de la región del Pacífico, a las extensiones de ésta en los valles del Río Magdalena y el bajo Río Cauca, y el Río Sinú, en el centro y Norte de Colombia. Además de los componentes andinopacífico y centro-americano compartidos con la costa pacífica, estos valles presentan más afinidades con la parte oriental de los Andes (Attalea butyracea) y un endemismo propio. En total se encuentran 40 especies por debajo de los $1000 \mathrm{~m}$ en los valles de Cauca y Magdalena.

La flora de palmeras del valle húmedo del Magdalena comparte 28 especies con el resto de la región del Pacífico, mientras que tres especies son endémicas de este valle: Geonoma chlamydostachys, Astrocaryum triandrum y Attalea nucifera. Attalea cohune, presente en el continente suramericano solo en un sector de este valle, representa una interesante disyunción entre esta región y el norte de Centroamérica, donde es abundante (Galeano \& Bernal, 2002).

Ciertas especies endémicas o sub-endémicas de estos valles no entran en el cálculo por ser compartidos con la zona andina (Sección 6), como Aiphanes parvifolia y A. simplex, o son tratadas como endemismos andinos por crecer por encima de los 1000 $\mathrm{m}$ (Attalea amygdalina).

\section{Los Andes, las cordilleras costeras caribeñas y las montañas guyanesas}

Géneros: 24; especies: 135 (1000-3500 m); endemismo: $39 \%$ (especies restringidas a la zona $1000-3500 \mathrm{~m}$ ).

Estas tres entidades, a pesar de incluir formaciones orogénicas muy diversas (cordilleras de cerca de 10 millones de años frente a las zonas de subducción de las placas pacíficas y de Nazca, con volcanismo activo, antiguas cordilleras de Cutucú, Cóndor y Campanquiz en la Amazonía occidental, formaciones precámbricas del escudo Guyanés), tienen una indiscutible afinidad florística. La presencia en conjunto de Dictyocaryum, Prestoea y de taxones del complejo de Geonoma undata, une a este grupo de montañas.

El límite de $1000 \mathrm{~m}$ escogido ha sido justificado a propósito del dominio amazónico (Sección 1), pero es necesariamente algo arbitrario. Entre los géneros tropicales que se encuentran esporádicamente por encima de los $1000 \mathrm{~m}$, podemos mencionar a Attalea, con tres especies que sobrepasan esta altitud, una de ellas (A. amygdalina) creciendo desde los $1000 \mathrm{~m}$ hacia arriba; en Astrocaryum, una sola especie sobrepasa los 1000 $\mathrm{m}$ y alcanza $1650 \mathrm{~m}$ ( $A$. faranae); en Oenocarpus, una especie sobrepasa los $1000 \mathrm{~m}$ también $(O$. bataua hasta $1400 \mathrm{~m}$ ). Los géneros Desmoncus, Mauritia y Mauritiella justo alcanzan el límite de los $1000 \mathrm{~m}$. Entre los géneros característicos de las altitudes superiores, Parajubaea no desciende por debajo de los $1500 \mathrm{~m}$, Ceroxylon está totalmente contenido por encima de los $1000 \mathrm{~m}$ a excepción de Ceroxylon amazonicum (800-1200 m) y de poblaciones marginales de C. echinulatum y C. parvum en el suroccidente de Ecuador alrededor de 800—900 m; Dictyocaryum está también por encima de los $1000 \mathrm{~m}$, a excepción de tres poblaciones aisladas en el occidente amazónico y muy raramente de $D$. Lamarkianum bajando los Andes hasta los 800 $\mathrm{m}$. Sin embargo la noción de endemismo montañoso depende en gran medida del límite altitudinal escogido. Por ejemplo, Prestoea simplicifolia, la cual crece entre 950 y 1600 m no está considerada dentro de los endemismos andinos (1000-3500 $\mathrm{m})$, porque es compartida con la región pacífica $(0-1000 \mathrm{~m})$. El valor de endemismo reportado de $39 \%$ es en consecuencia una subestimación de lo que se puede considerar realmente como el elemento montañoso, el cual podría subir hasta los $60 \%$ con la prolongación de rango altitudinal de varias especies en los pie-de-montes pacíficos, amazónicos y caribeños hasta los $400-800 \mathrm{~m}$.

\section{Los Andes}

Aunque las palmeras que crecen en los Andes han sido tratadas separadamente en el estudio de cada país andino (Kahn \& Moussa, 1994; Galeano \& Bernal, 1987; Wessesls Boer, 1988; Borchsenius et al., 1998; Stauffer, 1999; Moraes, 2004) y en la guía de palmas de América (Henderson et al., 1995), análisis más recientes de las palmeras de la región andina, en su conjunto, fueron realizados por Moraes et al. (1995) y Borschenius \& Moraes (2006).

Los Andes tropicales por encima de los $1000 \mathrm{~m}$ albergan al menos 121 especies de palmeras, agrupadas en 24 géneros. Los géneros más diversificados son Aiphanes (23 spp.), Geonoma (20 spp.), Wettinia (16 spp.) y Ceroxylon (11 spp.), que en conjunto, representan casi el $60 \%$ de las palmeras que crecen en la región andina. Exceptuando a Geonoma, que tiene una distribución geográfica y ecológica amplia en el Neotrópico, la diversificación de estos géneros ha estado determinada por los nuevos hábitats y las barreras establecidas con el levantamiento de los Andes. Los géneros Aiphanes y Wettinia, particularmente, aunque se han extendido hasta las tierras bajas, tanto en el Pacífico como en la Amazonía, con representación de endemismos notables en estas regiones, alcanzan su mayor diversidad en los Andes tropicales. Incluso géneros como Iriartea y Socratea, considerados elementos 
típicos de las tierras bajas amazónicas, probablemente tienen afinidades andinas (Malagón \& Bernal, 2002). Estos autores encontraron que en las vertientes amazónicas de los Andes en Colombia, la abundancia de Iriartea deltoidea aumenta a medida que se asciende en los Andes, y se hace máxima hacia los $1300 \mathrm{~m}$, donde la especie es reemplazada abruptamente por Dictyocaryum lamarckianum. Algo parecido sucede con Socratea exhorriza, que es reemplazada por $S$. rostrata hacia los $900 \mathrm{~m}$. Es interesante también el caso de Hyospathe, con cuatro especies endémicas de los Andes, entre las seis que componen el género.

Solo hay dos géneros exclusivos y casi exclusivos de los Andes: Parajubaea, con tres especies restringidas a elevaciones por encima de $1500 \mathrm{~m}$, y Ceroxylon, con 12 especies, y solo una o dos de ellas, descendiendo hasta elevaciones de $800 \mathrm{~m}$. A nivel específico, sin embargo, la situación es contrastante, pues 50 especies (41\% del total de palmas andinas) son exclusivas de esa región (por encima de los $1000 \mathrm{~m}$ ). Al igual que se ha propuesto para otras familias como las Rubiaceae (Andersson, 1995), también en palmeras el bajo endemismo a nivel genérico y alto a nivel específico podría interpretarse como el resultado de radiaciones adaptativas en tiempos recientes en el área de los Andes.

Otras contribuciones importantes a la diversidad de palmeras en los Andes tropicales incluyen géneros y especies propias de las tierras bajas, que ascienden en los Andes a elevaciones ligeramente superiores a $1000 \mathrm{~m}$; tal es el caso, entre otras, de Bactris (4 spp.), Attalea (3 spp., aunque una, A. amygdalina, es exclusivamente andina), Euterpe (3 spp., con una, E. luminosa, exclusivamente andina). Pholidostachys y Phytelephas (2 spp. cada uno), Syagrus (3 spp.)

El gradiente de riqueza en especies decrece a medida que se asciende en los Andes: el 75\% de las especies de toda la región se encuentra en la franja altitudinal de 1000 a $1500 \mathrm{~m}$, seguido por la franja de $1500-2000 \mathrm{~m}$ (60\%, 73 spp.), 28 especies entre $2000-2500 \mathrm{~m}$, y por encima de $3000 \mathrm{~m}$ de elevación se han registrado sólo cinco especies: Geonoma megalospatha, $G$. orbignyana, G. undata, Parajubaea torallyi y Ceroxylon parvifrons; esta última registrada hasta $3500 \mathrm{~m}$ en los Andes ecuatorianos (Borchsenius \& Moraes, 2006), la más alta elevación registrada para una palmera en los Andes y en el mundo.

La mayor concentración de especies de palmeras andinas se encuentra al norte de la región, en los Andes de Colombia (21 géneros, 80 especies), situación que no es de extrañar, dada la mayor complejidad topográfica y prevalencia de ambientes húmedos de esta parte de los Andes, comparada con el resto de la región. En los Andes de Ecuador se encuentran 65 especies en 18 géneros, y en los peruanos 45 especies en 18 géneros. La riqueza de palmeras disminuye hacia los extremos de los Andes, con 27 especies en 11 géneros en Venezuela, y 21 especies en 12 géneros en Bolivia.

La distribución de los endemismos en la región (restringidos a la zona superior a $1000 \mathrm{~m}$ ) sigue el mismo patrón de las palmeras andinas en general: 15 especies concentradas al norte, en los Andes colombianos, siete especies en los Andes ecuatorianos, seis especies en los Andes peruanos, cuatro especies en los Andes bolivianos (Syagrus yungasensis, Parajubaea torrallyi, P. sunkha y una especie no descrita de Ceroxylon) y una en los Andes venezolanos (Aiphanes stergiosii).

Las antiguas cordilleras de Cutucú, Condor y Campanquiz, en el sureste de Ecuador y norte de Perú, aunque geológicamente distintas de los Andes, presentan una flora de palmeras típicamente andino-amazónicas, con pocos endemismos (Wettinia minima).

En la distribución de las palmeras andinas, en su conjunto, se destaca la presencia de 11 especies (Ceroxylon parvifrons, $C$. vogelianum, Chamaedorea linearis, C. pinnatifrons, Dictyocaryum lamarckianum, Euterpe precatoria, Geonoma euspatha, G. interrupta, G. orbignyana, G. undata y Prestoea acuminata), de diferentes afinidades biogeográficas, pero que están presentes a todo lo largo de los Andes tropicales, desde Venezuela hasta Bolivia. Dentro de este grupo, se destacan Chamaedorea pinnatifrons y C. linearis, que se encuentran desde el nivel del mar, hasta 2700 y $2800 \mathrm{~m}$, respectivamente, mostrando la más amplia plasticidad en palmeras tropicales, en términos altitudinales. Bactris setulosa aparentemente tiene una distribución similar aunque terminando en el Perú central, pero podría ser en realidad un conjunto de dos especies crípticas no emparentadas (Couvreur et al. 2007).

\section{Las cordilleras costeras caribeñas}

Estas pequeñas cordilleras aisladas en la costa Caribe de Colombia (Sierra Nevada de Santa Marta) y de Venezuela son geológicamente distintas pero aparecen a nivel florístico como una prolongación de los Andes, con los cuales comparten algunas especies típicas (Bactris setulosa, Ceroxylon alpinum, C. ceriferum, Chamaedorea linearis, Dictyocaryum lamarkianum, Geonoma orbignyana, G. undata, Prestoea carderi y Wettinia praemorsa), pero tienen su endemismo propio (Asterogyne spp., Dictyocaryum fuscum, Geonoma spp.)

\section{Las montañas guyanesas}

Estas montañas de arenisca son muy antiguas (Precámbrico) y siempre han estado aisladas de los focos de especiación de los elementos montańosos neotropicales (América Central y Andes), de tal modo que solo la dispersión a larga distancia puede proveer especies adaptadas a las altitudes superiores. De hecho, solo nueve especies de palmeras crecen por encima de los $1000 \mathrm{~m}$ en las montañas guyanesas, entre las cuales se destacan las endémicas Dictyocaryum ptarianum y Prestoea tenuiramosa (Stauffer, 2000). Poblaciones de Dictyocaryum de la Amazonía occidental baja han sido atribuidas a $D$. ptarianum, pero eso es cuestionable porque además de crecer en un ámbito totalmente diferente y geográfícamente distante, estas poblaciones son cespitosas mientras que el típico D. ptarianum de las laderas y planicies superiores de los tepuyes es solitario. Los dos otros endemismos de las montañas guyanesas, Geonoma appuniana y G. fusca son probablemente mejor considerados como subespecies de $G$. undata y $G$. aspidiifolia, respectivamente (A. Henderson, en prep.).

\section{La vegetación costera}

La vegetación costera es variada a lo largo de las costas pacíficas y atlánticas de América del Sur. En muchos lugares, las palmeras se han adaptado a las condiciones litorales. En las peñas erosionadas del litoral de Esmeraldas (Ecuador), existen poblaciones de Aiphanes eggersii a pocos metros del mar. Esta especie crece más al sur adentro de las tierras en bosque seco, mientras que está limitada a la franja seca del litoral en Esmeraldas, a poca distancia del bosque húmedo del Chocó. Al extremo norte de Esmeraldas y en Colombia, existen densas poblaciones de Euterpe oleracea en los bosques inundables de los estuarios, 
muy alejadas de las formaciones dominadas por Euterpe oleracea de las costas de las Guyanas y de la boca del Amazonas.

En la costa caribeña, Cocothrinax barbadensis crece en lugares escarpados de las vertientes rocosas expuestas a los vientos marinos.

En el estuario del Río Amazonas, Raphia taedigera forma densas poblaciones en bosque pantanoso.

En la parte del litoral Atlántico brasileño que pertenece al dominio de la Mata Atlántica, existe la Restinga, bosque bajo o matorral establecido sobre antiguas dunas de arena. La flora de palmeras de restinga es muy particular e incluye Allagoptera arenaria, A. brevicalyx, Attalea funifera, A. humilis, Bactris glassmanii, B. horridispatha, B. soeiroana, Butia capitata var. odorata, Syagrus coronata y S. schizophylla. Algunas de estas especies crecen hasta en las dunas expuestas al mar.

\section{Conclusión}

A partir del panorama presentado, se puede concluir que el conocimiento sobre las palmeras de América del Sur aumentó mucho durante los últimos 25 años, en los aspectos de taxonomía, fitogeografía y ecología.

Muchos aspectos todavía merecen ser profundizados. Algunos géneros carecen de una revisión crítica: Attalea (especialmente en el grupo de Scheelea, ver Pintaud, este volumen), Acrocomia, Manicaria, Desmoncus y Lepidocaryum, el grupo de Socratea andino y caribeño, el grupo de Astrocaryum acaulescentes del Brasil central (ver Kahn 2008 en el presente volumen), entre otros. Nuevos inventarios de regiones poco recolectadas seguirán aportando información de nuevas especies y nuevos registros, mientras que la degradación cada vez más preocupante de los ambientes naturales seguirá alimentando las listas de especies amenazadas.

La transformación de los ecosistemas por las actividades humanas, el cambio climático, son nuevos retos para la investigación científica: ¿cuáles son los mecanismos de adaptabilidad de las palmeras a esas nuevas condiciones ecológicas? ¿Porqué algunas se vuelven plantas invasoras (Attalea spp.)? Los estudios actuales y futuros tienen que ser cada vez más integrativos y aprovechar nuevas herramientas como la genómica o la ecoinformática para responder a éstas y otras preguntas.

\section{Literatura citada}

Aguirre Z., R.Linares-Palomino \& L.P. Kvist. 2006. Woody species and vegetation formations in seasonally dry forests of Ecuador and Peru. Arnaldoa, 13: 324-350.

Anderson A.B., P. May \& M.J. Balick. 1991. The subsidy from Nature. Columbia University Press, New York.

Anderson L. 1995. Diversity and origins of Andean Rubiaceae. Pp. 441-450, en: Churchill, S., Balslev, H, Forero, E. \& J.L. Luteyn (eds.), Biodiversity and Conservation of Neotropical Montane Forests. New York Botanical Garden Press, New York.

Askew G.P., D.J. Moffatt, R.F. Montgomery \& P.L. Searl. 1970. Interrelationships of soils and vegetation in the savannaforest boundary zone of Northeastern Mato Grosso. Geographical Journal, 136: 371-376.

Asmussen C.B., J.Dransfield, V. Deickmann, A.S. Barfod, J.-C. Pintaud \& W. J. Baker. 2006. A new subfamily classification of the palm family (Arecaceae): Evidence from Plastid DNA. Botanical Journal of the Linnean Society, 151: 15-38.

Aublet J. 1775. Histoire des plantes de la Guiane française. 4 vols.
Didot, Paris.

Aubreville A. 1961. Etude écologique des principales formations végétales du Brésil, et contribution à la connaissance des forêts de l'Amazonie brésilienne. Masson, Paris.

Baker W.J., T. A. Hedderson \& J. Dransfield. 2000a. Molecular phylogenetics of subfamily Calamoideae (Palmae) based on nrDNA ITS and cpDNA rps16 intron sequence data. Molecular Phylogenetics and Evolution, 14: 195-217.

Baker W.J., J. Dransfield \& T. A. Hedderson. 2000b. Phylogeny, character evolution, and a new classification of the calamoid palms. Systematic Botany, 25: 297-322.

Balslev H. \& M. Moraes. 1989. Sinopsis de las palmeras de Bolivia. AAU Reports 20: 1-107.

Barbosa Rodrigues J. 1903. Sertum Palmarum Brasiliensium, ou relation des palmiers nouveaux du Brésil, découverts, décrits et dessinés d'après nature. Imprimerie Monnom, Bruxelles, 2 vol., 1: 1-140, 91 pl.; 2: 1-114, 83 pl.

Barcelos E., G. Second, F. Kahn, P. Amblard, P. Lebrun \& M. Seguin. 1999. Memoirs of the New York Botanical Garden, 83: 191-201.

Bernal R. 1989. Endangerment of Colombian palms. Principes, 33(3): $113-128$

Bernal R. \& G. Galeano. 2006. Endangerment of Colombian Palms (Arecaceae): change over 18 years. Botanical Journal of the Linnean Society, 151: 151-163.

Billotte N., A.-M. Risterucci, E. Barcelos, J.-L. Noyer, P. Amblard \& F.C. Baurens. 2001. Development, characterization, and across-taxa utility of oil palm (Elaeis guineensis Jacq.) microsatellite markers. Genome, 44: 413-425.

Bjorholm S., J.C. Svenning, W.J. Baker, F. Skov \& H. Balslev. 2006. Historical legacies in the geographical diversity patterns of New World Palms. (Arecaceae) subfamilies. Botanical Journal of the Linnean Society, 151: 113-125.

Bjorholm S., J.C. Svenning, F. Skov \& H. Balslev. 2005. Environmental and spatial controls of palm (Arecaceae) species richness across the Americas. Global Ecology and Biogeography, 14: 423-429.

Borchsenius F. 1997. Plant comunities of eastern Ecuador. Principes, 41: 93-99.

Borchsenius F., H. Balslev \& J.-C. Svenning, 2001. Two new species of Geonoma sect. Taenianthera (Arecaceae) from the western Amazon. Nordic Journal of Botany, 21: 341-347.

Borchsenius F., H. Borgtoft Pedersen \& H. Balslev. 1998. Manual of the palms of Ecuador. AAU Report 37, Aarhus University Press, Aarhus, and Pontificia Universidad Católica del Ecuador.

Borchsenius F. \& M. Moraes. 2006. Diversidad y usos de palmeras andinas (Arecaceae). Pp. 412-433, en: Moraes, M., Ø11gaard, B., Bochsenius, F. \& Balslev, H. (eds.), Botánica Económica de Los Andes Centrales. Universidad Mayor de San Andrés, La Paz.

Borchsenius F. \& F. Skov. 1999. Conservation status of palms (Arecaceae) in Ecuador. Acta Botanica Venezuelica, 22: 221-236.

Bremer K. 2000. Early Cretaceous lineages of monocot flowering plants. PNAS, 97: 4707-4711.

Cabrera A.L. \& A. Willink. 1973. Biogeografía de América latina. Programa regional de desarrollo científico y técnico, Secretaría General de la Organización de Estados Americanos, Washington, USA.

Candolle A. de. 1804. Mémoire sur le Ceroxylon, nouveau genre de palmiers. Bulletin des Sciences, par la Société Philomathique, Paris 49: 239-240.

Charles-Dominique P., J. Chave, M.-A. Dubois, de J.-J. Granville, B. Riera \& C. Vezzoli. 2003. Colonization front of the understorey palm Astrocaryum sciophilum in a pristine rain forest of French Guiana. Global Ecology and Bioge- 
ography, 12: 237-248.

Couvreur T.L.P., W.J. Hahn, J.-J. de Granville, J.-L. Pham, B. Ludeña \& J.-C. Pintaud. 2007. Phylogenetic relationships of the cultivated Neotropical palm Bactris gasipaes (Arecaceae) with its wild relatives inferred from chloroplast and nuclear DNA polymorphisms. Systematic Botany, 32(3): 519-530

Cuenca A. \& C.B. Asmussen. 2007. Phylogeny of the Palm Tribe Chamaedoreeae (Arecaceae) Based on Plastid DNA Sequences. Systematic Botany, 32: 250-263.

Cuenca A., C.B. Asmussen-Lange \& F. Borchsenius. 2008. A dated phylogeny of the palm tribe Chamaedoreeae supports Eocene dispersal between Africa, North and South America. Molecular Phylogenetics and Evolution, 46: 760-775.

Dransfield J., N.W. Uhl, C.B. Asmussen, W.J. Baker, M.M. Harley \& C.E. Lewis. 2005. A new phylogenetic classification of the palm family, Arecaceae. Kew Bulletin, 60: 559-569.

Drude O. 1881-1882. Palmae in Martius Flora Brasiliensis, 3: 253 460, 461-583.

Dugand A. 1940. Palmas de Colombia. Clave diagnostica de los géneros y nomina de las especies conocidas. Caldasia, 1: 20-83.

Eslava J. 1992. La precipitación en la región del Pacífico colombiano (Lloró: el sitio más lluvioso del mundo?). Zenit, 3: 7-33.

Eslava J. 1993. Climatología. Pp. 136-147, en: P. Leyva (ed.). Colombia Pacífico. Tomo I. Fondo FEN-COLOMBIA, Bogotá.

Espurt N., P. Baby, S. Brusset, M. Roddaz, W. Hermoza \& et al. 2007. How does the Nazca Ridge subduction influence the modern Amazonian foreland basin? Geology, 35: 515-518.

Forero E. y A. Gentry. 1989. Lista anotada de las plantas el Departamento del Chocó, Colombia. Biblioteca José Jerónimo Triana No. 10. Instituto de Ciencias Naturales, Universidad Nacional de Colombia, Bogotá.

Galeano G. 1991 - Las palmas de la región de Araracuara. Tropenbos, Bogotá.

Galeano G. 1992. Patrones de distribución de la palmas de Colombia. Bulletin de l'Institut français d'Études Andines, 21: 599-607.

Galeano G. 2001. Estructura, composición y riqueza del componente arbóreo de los bosques del Golfo de Tribugá, Chocó, Colombia. Caldasia, 23: 213-236.

Galeano G. \& R. Bernal, 1987. Las palmas del departamento de Antioquia. Región Occidental. Universidad Nacional de Colombia, Centro Editorial, Bogotá.

Galeano G. \& R. Bernal, 2002. New species and new records of Colombian palms. Caldasia, 24: 277-292.

Galeano G. \& R. Bernal. 2005. Palmas. Pp. 59-224, en: Calderón, E., G. Galeano \& N. García (eds.), Libro Rojo de Plantas de Colombia. Volumen II: Palmas, frailejones y zamias. Instituto Alexander von Humboldt, Instituto de Ciencias Naturales-Universidad Nacional de Colombia, Ministerio del Medio Ambiente. Bogotá, Colombia.

Gentry A. 1982. Phytogeographic patterns as evidence for a Chocó refuge. Pp. 112-136, in: G. T. Prance (ed.). Biological Diversification in the Tropics. New York.

Gentry A. 1986a. Notes on Peruvian palms. Annals of the Missouri Botanical Garden, 73: 158-165.

Gentry A. 1986b. Species richness and floristic composition of Chocó region plant communities. Caldasia, 15: 71-79.

Gentry A. 1993. Riqueza y composición florística. Pp. 200-219, en: P. Leyva (ed.). Colombia Pacífico. Tomo I. Fondo FENCOLOMBIA, Bogotá.

Glassman S. 1999. A taxonomic treatment of the palm subtribe Attaleinae. Illinois Biological Monographs 59. University of Illinois Press, Urbana.

Granville J.-J. de. 1997. Arecaceae. In: Guide to the Vascular Plants of Central French Guiana: Part 1. Pteridophytes, Gymnos- perms, and Monocotyledons. Memoirs of the New York Botanical Garden, Vol. 76.

Gunn B. 2004. The phylogeny of the Cocoeae (Arecaceae) with emphasis on Cocos nucifera. Annals of the Missouri Botanical Garden, 91: 505-522.

Haffer J. 1969. Speciation in Amazonian forest birds. Science, 165: 131-137.

Haffer J., 1970. Speciation in Colombian forest birds west of the Andes. Am. Mus. Novitates, 2294: 1-57.

Henderson A. 1995. The palms of the Amazon. Oxford University Press.

Henderson, A. 2000. Bactris (Palmae). Flora Neotropica Monograph 79. New York: The New York Botanical Garden.

Henderson A. \& M.J. Balick. 1987. Notes of the palms of Amazônia Legal. Principes 31: 116-122.

Henderson A., F. Borchsenius \& H. Balslev. 2008. New species of Geonoma (Palmae) from Ecuador. Brittonia 60: 190201.

Henderson A., G. Galeano \& R. Bernal. 1995. Field guide to the palms of the Americas. Princeton University Press.

Hooghiemstra H. 2002. The dynamic rainforest ecosystem on geological, quaternary and human time scales. In Verweij P. (ed.), Understanding and capturing the multiple values of tropical forests. Proceedings of the international seminar on valuation and innovative financing mechanisms in support of conservation and sustainable management of tropical forests, pp. 7-19.

Hooghiemstra H. \& T. van der Hammen. 1998. Neogene and Quaternary development of the neotropical rain forest: the forest refugia hypothesis, and a literature overview. Earth-Science Reviews, 44: 147-183.

Humboldt A. von, A. Bonpland \& C. Kunth. 1816. Nova Genera et Species Plantarum I. Libraisrie Grecque-Latine-Allemande, Paris.

Jacquin J. 1763. Selectarum Stirpium Americanarum Historia. Viena. (2nda ed. 1781).

Jaramillo C., M.J. Rueda \& G. Mora 2006. Cenozoic plant diversity in the Neotropics. Science, 311: 1893-1896.

Kahn F. 1997. The Palms of Eldorado. Orstom, The Internatonal Palm Society, Ed. Champflour, Marly-le-Roi, 252 p.

Kahn F. 2008. El género Astrocaryum (Arecaceae), Rev. peru. biol. 15(supl. 1): 031- 048

Kahn F. \& J.-J. de Granville. 1992. Palms in forest ecosystems of Amazonia. Springer Verlag, Berlin, $226 \mathrm{p}$.

Kahn F. \& K. Mejía. 1991. The palm communities of two terra firme forests in Peruvian Amazonia. Principes, 35: 22-26.

Kahn F. \& F. Moussa. 1994. Las palmeras del Perú - Colecciones, Patrones de distribución geográfica, Ecología, Estatus de conservación, Nombres vernáculos, Utilizaciones. IFEA, Lima, $180 \mathrm{p}$.

Karsten H. 1857. Plantae Columbianae. Linnaea, 28: 241-81, 387412.

Ledru M.-P., M.-L. Ferraz Salatino, G. Ceccantini, A. Salatino, F. Pinheiro \& J.-C. Pintaud. 2007. Regional assessment of the impact of climatic change on the distribution of a tropical conifer in the lowlands of South America. Diversity and Distributions, 13: 761-771.

MacBride J.F. 1960. Flora of Peru. Palmae. Part 1, n², Field Mus. Nat. Hist. Bot., 13 : 321-418.

Malagón W. \& R. Bernal. 2002. Distribución altitudinal de las palmas en la vertiente oriental de los Andes de Colombia. p. 209, en Rangel-Ch., J. O., J. Aguirre-C. \& M. G. Andrade-C. (eds.), Libro de Resúmenes Octavo Congreso Latinoamericano y Segundo Colombiano de Botánica. Instituto de Ciencias Naturales, Universidad Nacional de Colombia, Bogotá, Colombia.

Martius C.F.P. von, 1823-1853. Historia Naturalis Palmarum, 3 
Vol., Munich.

Martius C.F.P. von, 1844. Palmetum Orbignianum in . A. d'Orbigny, Voyage dans l'Amérique méridionale, 7 (3): 1-140, Paris.

Meyer G.F.W. 1818. Primitiae florae essequiboensis. H. Dieterich, Göttingen.

Millán B. 2006. Arecaceae endémicas del Perú. en: León, B. et al. (ed.), El Libro Rojo de las Plantas Endémicas del Perú. Rev. Peru. Biol., Número especial 13 (2): 706-707.

Montúfar R. \& J.-C. Pintaud. 2006. Variation in species composition, abundance and microhabitat preferences among Western Amazonian terra firme palm communities. Botanical Journal of the Linnean Society, 151: 127-140.

Montúfar R. 2007. Structure génétique, biochimique, morphologique et écologique de Oenocarpus bataua Mart. (Arecaceae): perspectives pour la valorisation durable d'une ressource forestière néotropicale. Thèse, Ecole Nationale Supérieure Agronomique de Montpellier.

Moore Jr. H.E. 1973. Palms in the tropical forest ecosystems of Africa and South America. In: Meggers B.J., Ayensu E.S. and Duckworth W.D. eds. Tropical forest ecosytems in Africa and South America: a comparative review. Smithsonian Institute Press, Washington.

Moraes M. 1999. Fitogeografía de palmeras en las tierras bajas de Bolivia. Acta Botanica Venezuelica, 22: 127-140.

Moraes M. 2004. Flora de palmeras de Bolivia. Plural Editores, La Paz.

Moraes M. 2007. Phytogeographical patterns of Bolivian palms. Palms, 51: 177-186.

Moraes M., G. Galeano, R. Bernal, H. Balslev \& A. Henderson. 1995. Tropical Andean palms. In: Churchill, S., Balslev, H, Forero, E. \& J.L. Luteyn (eds.), Biodiversity and Conservation of Neotropical Montane Forests. New York Botanical Garden Press, New York, pp. 473-488,

Moussa F., I.P.A. Miranda \& F. Kahn 1994. Palmeiras no herbário do INPA. INPA, Manaus, $94 \mathrm{p}$.

Noblick L. 2004a. Transfer of Syagrus campicola to Butia. Palms, 48: 42.

Noblick L. 2004b. Syagrus vermicularis, a fascinating new palm from Northern Brazil. Palms, 48: 109-116.

Noblick L.R. 2006. The grassy Butia: two new species and a new combination. Palms 50(4): 167-178.

Norup M.V., J. Dransfield, M.W. Chasse, A.S. Barfod, E.S. Fernando \& W.J. Baker. 2006. Homoplasious character combinations and generic delimitation: a case study from the Indo-Pacific arecoid palms (Arecoideae: Areceae). American Journal of Botany, 93: 1065-1080.

Oersted A.S. 1859. Palmae Centroamericanae. Naturhist. Foren. Vidensk. Meddelelser.

Pennington R.T. \& C.W. Dick. 2004. The role of immigrants in the assembly of the South American rainforest tree flora. Phil. Trans. R. Soc. Lond. B. 359, 1611-1622.

Pintaud J.-C. \& B. Millán. 2004. Notes on Chamaedorea in Peru. Palms, 48: 167-174.

Poux C., P.Chevret, D. Huchon, W.W. de Jong \& E.J.P. Douzery. 2006. Arrival and diversification of caviomorph rodents and platyrrhine primates in South America. Syst. Biol., 55: 228-244.

Prance G.T. 1973. Phytogeographic support for the theory of Pleistocene forest refuges in the Amazon basin, based on evidence from distribution patterns in Caryocaraceae, Chrysobalanaceae, Dichapetalaceae and Lecythidaceae. Acta Amazônica 3: 5-28.

Räsänen M., J. Salo \& R. Kalliola. 1987. Fluvial perturbance in the western Amazonian river basin: regulation by long-term sub-Andean tectonics. Science, 238: 1398-1401.

Räsänen M., J. Salo, H. Jungner \& L. Romero-Pitman. 1990. Evo- lution of the western Amazon relief: impact of Andean foreland dynamics. Terra Nova 2: 320-332.

Raven P.H. \& D.I. Axelrod. 1972. Plate tectonics and Australasian paleobiogeography. Science, 176: 1379-1386.

Renner S.S., 2004. Plant dispersal across the tropical Atlantic by wind and sea currents. Int. J. Plant Sci., 165 (4 Suppl.) S1-S11.

Rizzini C.T. 1963. A flora do cerrado. Analise floristica das savanas centrais. In: simposio sôbre o cerrado. Univ. São Paulo, pp. $125-177$

Ruiz H. \& J. Pavón. 1794. Flora Peruviana Prodromus. Madrid.

Ruiz H. \& J. Pavón. 1798. Systema Vegetabilium Florae Peruviana et Chilensis. Madrid.

Sampaio A.J. de. 1933. A zona dos cocais e sua individualização na fitogeografia do Brasil. Anais Acad. Brasil. Ciências, 5: 61-65.

Silva J.B.F. da \& C.R. Clement. 2005. Wild pejibaye (Bactris gasipaes Kunth var. chichagui) in Southeastern Amazonia. Acta bot. bras., 19: 281-284.

Spruce R. 1871. Palmae amazonicae, sive enumeratio palmarum in itinere suo per regiones americae aequatoriales lectarum. Jour. Linn. Soc., 11: 65-175.

Stauffer F. 1999. Datos preliminares para la actualización de la flora de palmas (Arecaceae) de Venezuela. Acta Botanica Venezuelica, 22: 77-107.

Stauffer F.W. 2007. Arecaceae. In: Stefano R., Aymard G. y Huber O. (eds.) Flora vascular de los llanos de Venezuela. FUDENA, Fundación Empresas Polar, FIBV, Caracas.

Stauffer F.W. \& J.G. Fariñas 2006. The identity of Attalea macrolepis (Burret) Wess. Boer (Arecaceae). Candollea, 61: 83-88.

Schnell R. 1987. La flore et la végétation de l'Amérique tropicale. 2 vol. Masson, Paris.

Steege H., Pitman N., Phillips O., Chave j., Sabatier D., Duque A., J.-F. Molino, M-F. Prévost, R. Spichiger, H. Castellanos, P. Hildebrand \& R. Vasquez. 2006. Continental-scales patterns of canopy tree composition and function across Amazonia. Nature, 443: 444-447.

Steyermark J.A. 1951. Botanical exploration in Venezuela. I. Feldiana Bot., 28: 1-242.

Trénel P., M.M. Hansen, S. Normand \& F. Borchsenius 2008. Landscape genetics, historical isolation and cross-Andean gene flow in the wax palm Ceroxylon echinulatum (Arecaceae). Molecular Ecology (en prensa).

Trénel P., M.H.G. Gustafsson, W.J. Baker, C.B. Asmussen-Lange, J. Dransfield \& F. Borchsenius 2007. Mid-Tertiary dispersal, not Gondwanan vicariance explains distribution patterns in the wax palm subfamily (Ceroxyloideae: Arecaceae). Molecular Phylogenetics and Evolution, 45: 272-288.

Uhl N.W. \& J. Dransfield. 1987. Genera Palmarum. Allen Press, Lawrence, Kansas, USA.

Wallace A.R. 1853. Palm trees of the Amazon and their uses. London, $129 \mathrm{p}$.

Wesselingh F.P., M.E. Räsänen, G. Irion, H.B. Vonhof, R. Kaandorp, W. Renema, L. Romero Pittman \& M. Gingras. 2002. Lake Pebas: a palaeocological reconstruction of a Miocene, long-lived lake complex in western Amazonia. Cainozoic Research 1: 35-81.

Wessels Boer J.G. 1965. The indigenous palms of Suriname. E.J. Brill, Leiden.

Wessels Boer J.G. 1988. Palmas indigenas de Venezuela. Pittieria, 17: 1-332.

Wilf P., N. Rubén Cúneo, K.R. Johnson, J.F. Hicks, Wing S.L. \& J.D. Obradovich. 2003. High plant diversity in Eocene South America: Evidence from Patagonia. Science, 300: 122-125. 
Apéndice 1. Lista de las palmeras de América del sur continental. CS = Argentina, Uruguay y Paraguay (Cono Sur); BR = Brasil; BC= Bolivia y Chile; PE = Perú; EC = Ecuador; $C O$ = Colombia; VE = Venezuela; GU = Guyana, Suriname y Guyana Francesa. 1. La región amazónica, 2. Las formaciones de la periferia noroeste de la Amazonía y de la costa caribeña, 3. Las formaciones mesófilas y xéricas de la periferia Sur y Sur-Este de la Amazonía y del Cono Sur, 4. La Mata Atlántica, 5. La costa pacífica, el pie-de-monte andino occidental, la zona del istmo de Panamá y los valles interandinos de Colombia, 6 . Los Andes, las cordilleras costeras caribeñas y las montañas guyanesas, 7 . La vegetación costera. ?= población geográficamente aislada, tentativamente asignada a la especie correspondiente, o de presencia altamente probable pero no sustenciada por una colección de herbario. Cocos nucifera, cuya distribución natural es incierta, no está incluido en el listado, tampoco Attalea amylacea, A. fairchildensis, A. leandroana y A. lauromuelleriana descritos y conocidos solo en jardines botánicos.

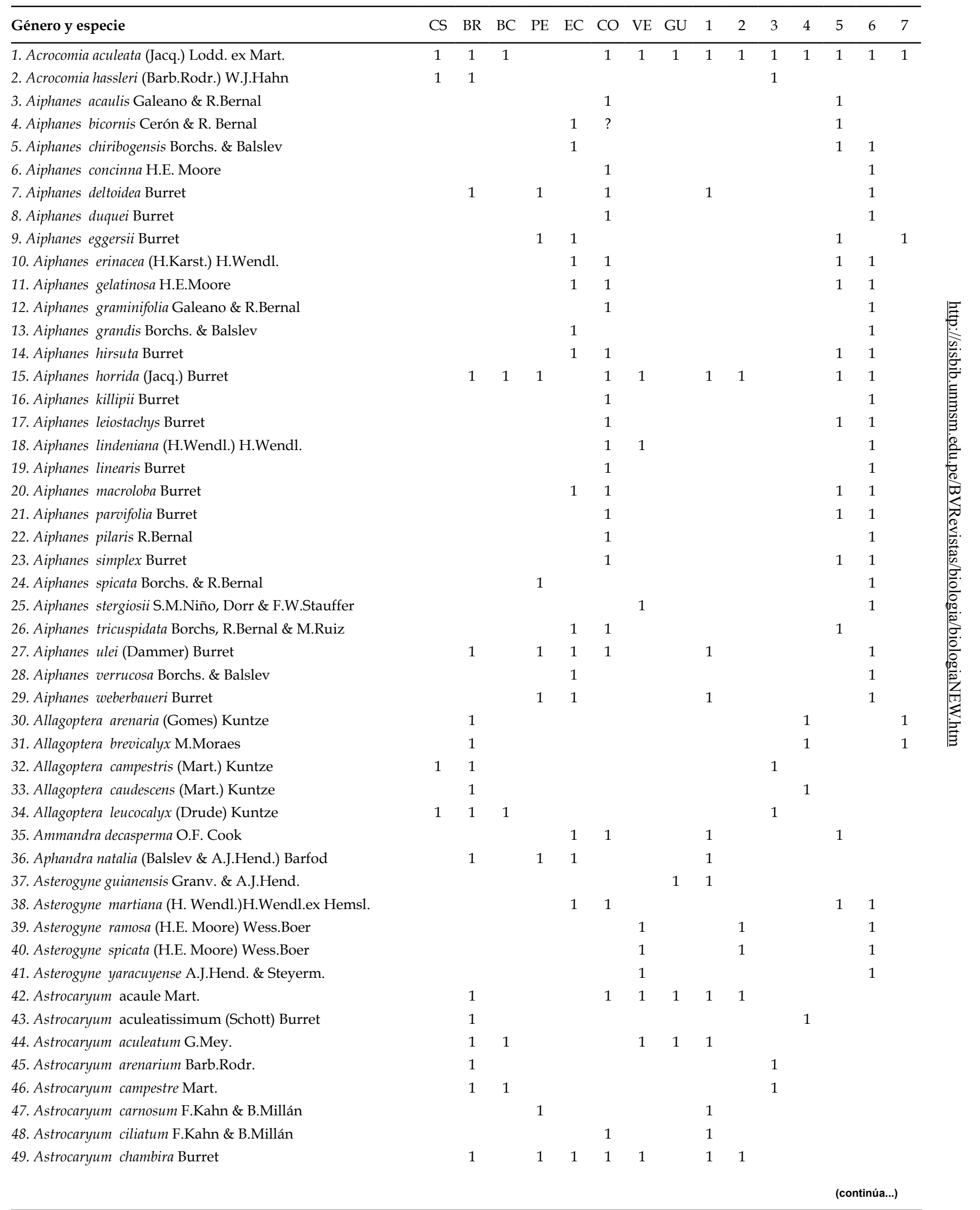


Apéndice 1. (continuación)

\begin{tabular}{|c|c|c|c|c|c|c|c|c|c|c|c|c|c|c|c|}
\hline Género y especie & CS & $\mathrm{BR}$ & $\mathrm{BC}$ & PE & EC & $\mathrm{CO}$ & VE & GU & 1 & 2 & 3 & 4 & 5 & 6 & 7 \\
\hline 50. Astrocaryum chonta Mart. & & 1 & 1 & 1 & & & & & 1 & & 1 & & & & \\
\hline 51. Astrocaryum cuatrecasanum Dugand & & & & & & 1 & & & 1 & & & & & & \\
\hline 52. Astrocaryum echinatum Barb.Rodr. & & 1 & & & & & & & & & 1 & & & & \\
\hline 53. Astrocaryum faranae F.Kahn \& E.Ferreira & & 1 & & 1 & & & & & 1 & & & & & 1 & \\
\hline 54. Astrocaryum farinosum Barb.Rodr. & & 1 & & & & & & 1 & 1 & & & & & & \\
\hline 55. Astrocaryum ferrugineum F.Kahn \& B.Millán & & 1 & & & & 1 & & & 1 & & & & & & \\
\hline 56. Astrocaryum giganteum Barb.Rodr. & & 1 & & & & & & & 1 & & & & & & \\
\hline 57. Astrocaryum gratum F.Kahn \& B.Millán & & & 1 & 1 & & & & & 1 & & 1 & & & & \\
\hline 58. Astrocaryum gynacanthum Mart. & & 1 & 1 & 1 & & 1 & 1 & 1 & 1 & 1 & & & & & \\
\hline 59. Astrocaryum huaimi Mart. & & 1 & 1 & 1 & & & & & & & 1 & & & & \\
\hline 60. Astrocaryum huicungo Dammer ex Burret & & & & 1 & & & & & 1 & & & & & & \\
\hline 61. Astrocaryum jauari Mart. & & 1 & 1 & 1 & 1 & 1 & 1 & 1 & 1 & 1 & & & & & \\
\hline 62. Astrocaryum javarense (Trail) Drude & & 1 & & 1 & & & & & 1 & & & & & & \\
\hline 63. Astrocaryum kewense Barb.Rodr. & & 1 & & & & & & & & & 1 & & & & \\
\hline 64. Astrocaryum macrocalyx Burret & & & & 1 & & 1 & & & 1 & & & & & & \\
\hline 65. Astrocaryum malybo H.Karst. & & & & & & 1 & & & & 1 & & & 1 & & \\
\hline 66. Astrocaryum minus Trail & & 1 & & & & & & 1 & 1 & & & & & & \\
\hline 67. Astrocaryum murumuru Mart. & & 1 & & & & & 1 & 1 & 1 & & & & & & \\
\hline 68. Astrocaryum paramaca Mart. & & 1 & & & & & & 1 & 1 & & & & & & \\
\hline 69. Astrocaryum perangustatum F.Kahn \& B.Millán & & & & 1 & & & & & 1 & & & & & & \\
\hline 70. Astrocaryum pygmaeum Drude & & 1 & & & & & & & & & 1 & & & & \\
\hline 71. Astrocaryum rodriguesii Trail & & 1 & & & & & & 1 & 1 & & & & & & \\
\hline 72. Astrocaryum sciophilum (Miq.) Pulle & & 1 & & & & & & 1 & 1 & & & & & & \\
\hline 73. Astrocaryum scopatum F.Kahn \& B.Millán & & & & 1 & & & & & 1 & & & & & & \\
\hline 74. Astrocaryum sociale Barb.Rodr. & & 1 & & & & & & & 1 & & & & & & \\
\hline 75. Astrocaryum standleyanum L.H.Bailey & & & & & 1 & 1 & & & & & & & 1 & & \\
\hline 76. Astrocaryum triandrum Galeano,R.Bernal \& F.Kahn & & & & & & 1 & & & & & & & 1 & & \\
\hline
\end{tabular}

77. Astrocaryum ulei Burret

78. Astrocaryum urostachys Burret

79. Astrocaryum vulgare Mart.

80. Astrocaryum weddelii Drude

81. Attalea allenii H.E.Moore

82. Attalea amygdalina Kunth

83. Attalea anisitsiana (Barb.Rodr.) Zona

84. Attalea apoda Burret

85. Attalea attaleoides (Barb.Rodr.) Wess.Boer

86. Attalea barreirensis Glassman

87. Attalea bassleriana (Burret) Zona

88. Attalea brasiliensis Glassman

89. Attalea brejinhoensis (Glassman) Zona

90. Attalea burretiana Bondar

91. Attalea butyracea (Mutis ex L.f) Wess.Boer

92. Attalea camopiensis (Glassman) Zona

93. Attalea cephalotus Poepp. ex Mart.

94. Attalea cohune Mart.

95. Attalea colenda (O.F. Cook) Balslev \& A.J.Hend

96. Attalea compta Mart.

97. Attalea cuatrecasana(Dugand)Hend.Galeano Bernal

98. Attalea dahlgreniana (Bondar) Wess.Boer

99. Attalea degranvillei (Glassman) Zona

100. Attalea dubia (Mart.) Burret

101. Attalea eichleri (Drude) A.J.Hend.

102. Attalea exigua Drude

103. Attalea ferruginea Burret

104. Attalea funifera Mart.

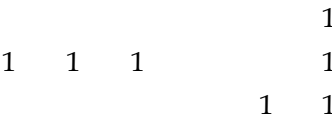


Apéndice 1. (continuación)

\begin{tabular}{|c|c|c|c|c|c|c|c|c|c|c|c|c|c|c|c|}
\hline Género y especie & CS & $\mathrm{BR}$ & $\mathrm{BC}$ & PE & EC & $\mathrm{CO}$ & VE & GU & 1 & 2 & 3 & 4 & 5 & 6 & 7 \\
\hline 105. Attalea geraensis Barb.Rodr. & & 1 & & & & & & & & & 1 & & & & \\
\hline 106. Attalea guaranitica Barb.Rodr. & 1 & & & & & & & & & & 1 & & & & \\
\hline 107. Attalea guianensis (Glassman) Zona & & & & & & & & 1 & 1 & & & & & & \\
\hline 108. Attalea humilis Mart. ex Spreng. & & 1 & & & & & & & & & & 1 & & & 1 \\
\hline 109. Attalea insignis (Mart.) Drude & & 1 & & 1 & 1 & 1 & & & 1 & 1 & & & & & \\
\hline 110. Attalea kewensis (Hook.f.) Zona & & & & $?$ & & & & & $?$ & & & & & & \\
\hline 111. Attalea luetzelburgii (Burret) Wess.Boer & & 1 & & & & 1 & 1 & & 1 & 1 & & & & & \\
\hline 112. Attalea macrocarpa (H.Karst.) Wess.Boer & & & & & & & 1 & & & 1 & & & & & \\
\hline 113. Attalea macrolepis (Burret) Wess.Boer & & & & & & $?$ & 1 & & & 1 & & & & & \\
\hline 114. Attalea maracaibensis Mart. & & & & & & 1 & 1 & & & 1 & & & & & \\
\hline 115. Attalea maripa (Aubl.) Mart. & & 1 & 1 & 1 & 1 & 1 & 1 & 1 & 1 & 1 & & & & & \\
\hline 116. Attalea maripensis (Glassman) Zona & & & & & & & & 1 & 1 & & & & & & \\
\hline 117. Attalea moorei (Glassman) Zona & & & & 1 & & & & & 1 & & & & & & \\
\hline 118. Attalea nucifera H.Karst. & & & & & & 1 & & & & & & & 1 & & \\
\hline 119. Attalea oleifera Barb.Rodr. & & 1 & & & & & & & & & & 1 & & & \\
\hline 120. Attalea osmantha (Barb.Rodr.) Wess.Boer & & & & & & & 1 & & & 1 & & & & & \\
\hline 121. Attalea peruviana Zona & & & & 1 & & & & & 1 & & & & & & \\
\hline 122. Attalea phalerata Mart. ex Spreng. & 1 & 1 & 1 & 1 & & 1 & & & 1 & & 1 & & & 1 & \\
\hline 123. Attalea pindobassu Bondar & & 1 & & & & & & & & & & 1 & & & \\
\hline 124. Attalea plowmanii (Glassman) Zona & & & & 1 & & 1 & & & 1 & & & & & & \\
\hline 125. Attalea polysticha (Burret) Wess.Boer & & 1 & & 1 & & 1 & 1 & 1 & 1 & & & & & & \\
\hline 126. Attalea princeps Mart. & & & 1 & & & & & & & & 1 & & & & \\
\hline 127. Attalea sagotii (Trail ex Thurn) Wess.Boer & & 1 & & & & & & 1 & 1 & & & & & & \\
\hline 128. Attalea salazarii (Glasman) Zona & & & & 1 & & & & & 1 & & & & & & \\
\hline 129. Attalea seabrensis Glassman & & 1 & & & & & & & & & & 1 & & & \\
\hline 130. Attalea septuagenata Dugand & & & & & & 1 & & & 1 & & & & & & \\
\hline 131. Attalea speciosa Mart. & & 1 & 1 & & & & & 1 & 1 & & 1 & & & & \\
\hline 132. Attalea spectabilis Mart. & & 1 & & & & & & 1 & 1 & & & & & & \\
\hline 133. Attalea tessmannii Burret & & 1 & & 1 & & & & & 1 & & & & & & \\
\hline 134. Attalea vitrivir Zona & & 1 & & & & & & & & & 1 & & & & \\
\hline 135. Attalea weberbaueri (Burret) Zona & & & & 1 & & & & & 1 & & & & & 1 & \\
\hline 136. Attalea wesselsboeri (Glassman) Zona & & & & & & & 1 & & & 1 & & & & & \\
\hline 137. Bactris acanthocarpa Mart. & & 1 & 1 & 1 & 1 & 1 & 1 & 1 & 1 & & & 1 & & & \\
\hline 138. Bactris acanthocarpoides Barb.Rodr. & & 1 & & & & & & 1 & 1 & & & & & & \\
\hline 139. Bactris aubletiana Trail & & & & & & & & 1 & 1 & & & & & & \\
\hline 140. Bactris bahiensis Noblick ex A.J.Hend & & 1 & & & & & & & & & & 1 & & & \\
\hline 141. Bactris balanophora Spruce & & 1 & & & & 1 & 1 & & 1 & & & & & & \\
\hline 142. Bactris barronis L.H. Bailey & & & & & & 1 & & & & & & & 1 & & \\
\hline 143. Bactris bidentula Spruce & & 1 & & 1 & & 1 & 1 & & 1 & & & & & & \\
\hline 144. Bactris bifida Mart. & & 1 & & 1 & & 1 & & & 1 & & & & & & \\
\hline 145. Bactris brongniartii Mart. & & 1 & 1 & 1 & & 1 & 1 & 1 & 1 & 1 & & & 1 & & \\
\hline 146. Bactris campestris Poepp. & & 1 & & & & 1 & 1 & 1 & 1 & 1 & & & & & \\
\hline 147. Bactris caryotifolia Mart. & & 1 & & & & & & & & & & 1 & & & \\
\hline 148. Bactris chaveziae A.J. Hend & & 1 & 1 & 1 & & & & & 1 & & & & & & \\
\hline 149. Bactris coloniata L.H. Bailey & & & & 1 & 1 & 1 & & & 1 & & & & 1 & & \\
\hline 150. Bactris coloradonis L.H. Bailey & & & & & 1 & 1 & & & & & & & 1 & & \\
\hline 151. Bactris concinna Mart. & & 1 & 1 & 1 & 1 & 1 & & & 1 & & & & & & \\
\hline 152. Bactris constanciae Barb.Rodr. & & 1 & & & & & & 1 & 1 & & & & & & \\
\hline 153. Bactris corossilla H.Karst. & & 1 & 1 & 1 & 1 & 1 & 1 & & 1 & 1 & & & & 1 & \\
\hline 154. Bactris cuspidata Mart. & & 1 & & & & & & 1 & 1 & & & & & & \\
\hline 155. Bactris elegans Barb.Rodr. & & 1 & 1 & & & 1 & & 1 & 1 & & & & & & \\
\hline 156. Bactris faucium Mart. & & & 1 & & & & & & 1 & & & & & & \\
\hline 157. Bactris ferruginea Burret & & 1 & & & & & & & & & & 1 & & & \\
\hline 158. Bactris fissifrons Mart. & & 1 & & 1 & 1 & 1 & & & 1 & & & & & & \\
\hline
\end{tabular}




\begin{tabular}{|c|c|c|c|c|c|c|c|c|c|c|c|c|c|c|c|}
\hline Género y especie & CS & BR & $\mathrm{BC}$ & $\mathrm{PE}$ & EC & $\mathrm{CO}$ & $\mathrm{VE}$ & GU & 1 & 2 & 3 & 4 & 5 & 6 & 7 \\
\hline 159. Bactris gasipaes Kunth & & 1 & 1 & 1 & 1 & 1 & 1 & & 1 & 1 & 1 & & 1 & 1 & \\
\hline 160. Bactris gastoniana Barb.Rodr. & & 1 & & & & & & 1 & 1 & & & & & & \\
\hline 161. Bactris glandulosa Oerst. & & & & & & 1 & & & & & & & 1 & & \\
\hline 162. Bactris glassmanii Med.Costa \& Noblick ex A.J.Hend & & 1 & & & & & & & & & & 1 & & & 1 \\
\hline 163. Bactris glaucescens Drude & 1 & 1 & 1 & & & & & & & & 1 & $?$ & & & \\
\hline 164. Bactris guineensis (L.) H.E. Moore & & & & & & 1 & 1 & & & 1 & & & & & \\
\hline 165. Bactris halmoorei A.J.Hend. & & 1 & & 1 & & & & & 1 & & & & & & \\
\hline 166. Bactris hatschbachii Noblick ex A.J.Hend & & 1 & & & & & & & & & & 1 & & & \\
\hline 167. Bactris hirta Mart. & & 1 & 1 & 1 & 1 & 1 & 1 & 1 & 1 & & & 1 & & & \\
\hline 168. Bactris hondurensis Standl. & & & & & 1 & 1 & & & & & & & 1 & & \\
\hline 169. Bactris horridispatha Noblick ex A.J.Hend & & 1 & & & & & & & & & & 1 & & & 1 \\
\hline 170. Bactris killipii Burret & & 1 & & 1 & & 1 & & & 1 & & & & & & \\
\hline 171. Bactris macroacantha Mart. & & 1 & 1 & 1 & & 1 & & & 1 & & & & & & \\
\hline 172. Bactris major Jacq. & & 1 & 1 & 1 & & 1 & 1 & 1 & 1 & 1 & 1 & & & & \\
\hline 173. Bactris maraja Mart. & & 1 & 1 & 1 & 1 & 1 & 1 & 1 & 1 & 1 & 1 & & 1 & 1 & \\
\hline 174. Bactris martiana A.J.Hend. & & 1 & & 1 & 1 & 1 & & & 1 & & & & & & \\
\hline 175. Bactris oligocarpa Barb.Rodr. & & 1 & & & & & & 1 & 1 & & & & & & \\
\hline 176. Bactris oligoclada Burret & & & & & & & 1 & 1 & 1 & & & & & & \\
\hline 177. Bactris pickelii Burret & & 1 & & & & & & & & & & 1 & & & \\
\hline 178. Bactris pilosa H.Karst. & & & & & 1 & 1 & 1 & & & 1 & & & 1 & & \\
\hline 179. Bactris pliniana Granv. \& A.J.Hend. & & 1 & & & & & & 1 & 1 & & & & & & \\
\hline 180. Bactris ptariana Steyerm. & & $?$ & & & & & 1 & 1 & 1 & & & & & 1 & \\
\hline 181. Bactris raphidacantha Wess.Boer & & 1 & & & & & & 1 & 1 & & & & & & \\
\hline 182. Bactris riparia Mart. & & 1 & 1 & 1 & 1 & 1 & & & 1 & & & & & & \\
\hline 183. Bactris rostrata Galeano \& R.Bernal & & & & & & 1 & & & & & & & 1 & & \\
\hline 184. Bactris schultesii (L.H.Bailey) Glassman & & & & 1 & 1 & & & & 1 & & & & & & \\
\hline 185. Bactris setiflora Burret & & & & & 1 & & & & 1 & & & & & & \\
\hline 186. Bactris setosa Mart. & & 1 & & & & & & & & & 1 & 1 & & & \\
\hline 187. Bactris setulosa H.Karst. & & & & 1 & 1 & 1 & 1 & & & 1 & & & 1 & 1 & \\
\hline 188. Bactris simplicifrons Mart. & & 1 & 1 & 1 & 1 & 1 & 1 & 1 & 1 & 1 & & & 1 & & \\
\hline 189. Bactris soeiroana Noblick ex A.J.Hend. & & 1 & & & & & & & & & & 1 & & & 1 \\
\hline 190. Bactris sphaerocarpa Trail & & 1 & & 1 & & 1 & & & 1 & & & & & & \\
\hline 191. Bactris syagroides Barb.Rodr. & & 1 & & & & & & & 1 & & & & & & \\
\hline 192. Bactris tefensis A.J.Hend. & & 1 & & & & & & & 1 & & & & & & \\
\hline 193. Bactris timbuiensis H.Q.B.Fern. & & 1 & & & & & & & & & & 1 & & & \\
\hline 194. Bactris tomentosa Mart. & & 1 & & & & & & 1 & 1 & & & & & & \\
\hline 195. Bactris turbinocarpa Barb.Rodr. & & 1 & & & & & & 1 & 1 & & & & & & \\
\hline 196. Bactris vulgaris Barb.Rodr. & & 1 & & & & & & & & & & 1 & & & \\
\hline 197. Barcella odora (Trail) Drude & & 1 & & & & & & & 1 & & & & & & \\
\hline 198. Butia archeri (Glassman) Glassman & & 1 & & & & & & & & & 1 & & & & \\
\hline 199. Butia arenicola (Barb.Rodr.) Burret & 1 & & & & & & & & & & 1 & & & & \\
\hline 200. Butia campicola (Barb.Rodr.) Noblick & 1 & & & & & & & & & & 1 & & & & \\
\hline 201. Butia capitata (Mart.) Becc. & 1 & 1 & & & & & & & & & 1 & 1 & & & 1 \\
\hline 202. Butia eriospatha (Mart. ex Drude) Becc. & & 1 & & & & & & & & & 1 & 1 & & & \\
\hline 203. Butia exospadix Noblick & 1 & 1 & & & & & & & & & 1 & & & & \\
\hline 204. Butia lallemantii Deble \& Marchiori & 1 & 1 & & & & & & & & & 1 & & & & \\
\hline 205. Butia leptospatha (Burret) Noblick & 1 & 1 & & & & & & & & & 1 & & & & \\
\hline 206. Butia marmorii Noblick & 1 & & & & & & & & & & 1 & & & & \\
\hline 207. Butia microspadix Burret & & 1 & & & & & & & & & 1 & & & & \\
\hline 208. Butia paraguayensis (Barb.Rodr.) L.H.Bailey & 1 & 1 & & & & & & & & & 1 & & & & \\
\hline 209. Butia purpurascens Glassman & & 1 & & & & & & & & & 1 & & & & \\
\hline 210. Butia yatay (Mart.) Becc. & 1 & 1 & & & & & & & & & 1 & & & & \\
\hline 211. Calyptrogyne baudensis A.J.Hend. & & & & & & 1 & & & & & & & 1 & & \\
\hline 212. Calyptrogyne costatifrons (L.H.Bailey) Nevers & & & & & & 1 & & & & & & & 1 & & \\
\hline 213. Ceroxylon alpinum Bonpl. ex DC. & & & & & & 1 & 1 & & & & & & & 1 & \\
\hline
\end{tabular}


Apéndice 1. (continuación)

\begin{tabular}{|c|c|c|c|c|c|c|c|c|c|c|c|c|c|c|c|}
\hline Género y especie & CS & $\mathrm{BR}$ & $\mathrm{BC}$ & PE & $\mathrm{EC}$ & $\mathrm{CO}$ & $\mathrm{VE}$ & GU & 1 & 2 & 3 & 4 & 5 & 6 & 7 \\
\hline 214. Ceroxylon amazonicum Galeano & & & & & 1 & & & & 1 & & & & & 1 & \\
\hline 215. Ceroxylon ceriferum (H.Karst.) Pittier & & & & & & 1 & 1 & & & & & & & 1 & \\
\hline 216. Ceroxylon echinulatum Galeano & & & & 1 & 1 & & & & & & & & 1 & 1 & \\
\hline 217. Ceroxylon parvifrons (Engel) H.Wendl. & & & 1 & 1 & 1 & 1 & 1 & & & & & & & 1 & \\
\hline 218. Ceroxylon parvum Galeano & & & 1 & 1 & 1 & & & & & & & & 1 & 1 & \\
\hline 219. Ceroxylon quindiuense (H.Karst.) H.Wendl. & & & & 1 & & 1 & & & & & & & & 1 & \\
\hline 220. Ceroxylon sasaimae Galeano & & & & & & 1 & & & & & & & & 1 & \\
\hline 221. Ceroxylon ventricosum Burret & & & & & 1 & 1 & & & & & & & & 1 & \\
\hline 222. Ceroxylon vogelianum (Engel) H.Wendl. & & & 1 & 1 & 1 & 1 & 1 & & & & & & & 1 & \\
\hline 223. Ceroxylon weberbaueri Burret & & & & 1 & & & & & & & & & & 1 & \\
\hline 224. Chamaedorea allenii L.H.Bailey & & & & & & 1 & & & & & & & 1 & 1 & \\
\hline 225. Chamaedorea angustisecta Burret & & 1 & 1 & 1 & & & & & 1 & & & & & & \\
\hline 226. Chamaedorea christinae Hodel & & & & & & 1 & & & & & & & 1 & & \\
\hline 227. Chamaedorea fragrans Mart. & & & & 1 & & & & & 1 & & & & & & \\
\hline 228. Chamaedorea linearis (Ruiz \& Pav.) Mart. & & & 1 & 1 & 1 & 1 & 1 & & & 1 & & & 1 & 1 & \\
\hline 229. Chamaedorea pauciflora Mart. & & 1 & 1 & 1 & 1 & 1 & & & 1 & & & & & & \\
\hline 230. Chamaedorea pinnatifrons (Jacq.) Oerst. & & 1 & 1 & 1 & 1 & 1 & 1 & & 1 & 1 & & & 1 & 1 & \\
\hline 231. Chamaedorea pygmaea H.Wendl. & & & & & & 1 & & & & & & & & 1 & \\
\hline 232. Chamaedorea ricardoi R.Bernal, Galeano \& Hodel & & & & & & 1 & & & & & & & 1 & & \\
\hline 233. Chamaedorea sullivaniorum Hodel \& N.W.Uhl & & & & & & 1 & & & & & & & 1 & 1 & \\
\hline 234. Chamaedorea tepejilote Liebm. & & & & & & 1 & & & & & & & 1 & 1 & \\
\hline 235. Chamaedorea warscewiczii H.Wendl. & & & & & 1 & 1 & & & & & & & 1 & 1 & \\
\hline 236. Chamaedorea woodsoniana & & & & & & 1 & & & & & & & & 1 & \\
\hline 237. Chelyocarpus chuco (Mart.) H.E.Moore & & 1 & 1 & & & & & & 1 & & & & & & \\
\hline 238. Chelyocarpus dianeurus (Burret) H.E. Moore & & & & & & 1 & & & & & & & 1 & & \\
\hline 239. Chelyocarpus repens F.Kahn \& K.Mejía & & & & 1 & & 1 & & & 1 & & & & & & \\
\hline 240. Chelyocarpus ulei Dammer & & 1 & & 1 & 1 & 1 & & & 1 & & & & & & \\
\hline 241. Cocothrinax barbadensis (Lodd. ex Mart.) Becc. & & & & & & & 1 & & & 1 & & & & & 1 \\
\hline 242. Copernicia alba Morong ex Morong \& Britton & 1 & 1 & 1 & & & & & & & & 1 & & & & \\
\hline 243. Copernicia prunifera (Mill.) H.E. Moore & & 1 & & & & & & & & & 1 & & & & \\
\hline 244. Copernicia tectorum (Kunth) Mart. & & & & & & 1 & 1 & & & 1 & & & & & \\
\hline 245. Cryosophila kalbreyeri (Dammer ex Burret) Dahlgren & & & & & & 1 & & & & 1 & & & 1 & 1 & \\
\hline 246. Cryosophila macrocarpa R.Evans & & & & & & 1 & & & & & & & 1 & & \\
\hline 247. Desmoncus cirrhiferus A.H.Gentry \& Zardini & & & & & 1 & 1 & & & & & & & 1 & & \\
\hline 248. Desmoncus giganteus A.J.Hend. & & 1 & 1 & 1 & 1 & 1 & & & 1 & & & & & & \\
\hline 249. Desmoncus mitis Mart. & & 1 & 1 & 1 & 1 & 1 & 1 & & 1 & & & & & & \\
\hline 250. Desmoncus orthacanthos Mart. & & 1 & 1 & 1 & 1 & 1 & 1 & 1 & 1 & 1 & 1 & & 1 & & \\
\hline 251. Desmoncus phoenicocarpus Barb.Rodr. & & 1 & 1 & & & & 1 & 1 & 1 & & & & & & \\
\hline 252. Desmoncus polyacanthos Mart. & & 1 & 1 & 1 & 1 & 1 & 1 & 1 & 1 & 1 & 1 & & & & \\
\hline 253. Dictyocaryum fuscum (H.Karst.) H.Wendl. & & & & & & & 1 & & & & & & & 1 & \\
\hline 254. Dictyocaryum lamarckianum (Mart.) H.Wendl. & & & 1 & 1 & 1 & 1 & 1 & & & & & & & 1 & \\
\hline 255. Dictyocaryum ptarianum (Steyerm.) H.E.Moore & & $?$ & & $?$ & & $?$ & 1 & 1 & $?$ & & & & & 1 & \\
\hline 256. Elaeis oleifera (Kunth) Cortés & & 1 & & 1 & 1 & 1 & & 1 & 1 & 1 & & & 1 & & \\
\hline 257. Euterpe catinga Wallace & & 1 & & 1 & 1 & 1 & 1 & 1 & 1 & & & & & 1 & \\
\hline 258. Euterpe edulis Mart. & 1 & 1 & & & & & & & & & & 1 & & & \\
\hline 259. Euterpe espiritosantensis Fernandes & & 1 & & & & & & & & & & 1 & & & \\
\hline 260. Euterpe longibracteata Barb.Rodr. & & 1 & & & & & 1 & 1 & 1 & & & & & & \\
\hline 261. Euterpe luminosa A.J.Hend., Galeano \& Meza & & & & 1 & & & & & & & & & & 1 & \\
\hline 262. Euterpe oleracea Mart. & & 1 & & & 1 & 1 & 1 & 1 & 1 & 1 & & & 1 & & 1 \\
\hline 263. Euterpe precatoria Mart. & & 1 & 1 & 1 & 1 & 1 & 1 & 1 & 1 & 1 & & & 1 & 1 & \\
\hline 264. Geonoma appuniana Spruce & & 1 & & & & & 1 & 1 & & & & & & 1 & \\
\hline 265. Geonoma aspidiifolia Spruce & & 1 & & & & & & & 1 & & & & & & \\
\hline 266. Geonoma atrovirens Borchs. \& Balslev & & & & 1 & 1 & 1 & & & 1 & & & & & & \\
\hline 267. Geonoma awensis A.J.Hend., Borchs. \& Balslev & & & & & 1 & 1 & & & & & & & 1 & & \\
\hline 268. Geonoma baculifera (Poit.) Kunth & & 1 & & & & $?$ & 1 & 1 & 1 & & & & & & \\
\hline
\end{tabular}




\begin{tabular}{|c|c|c|c|c|c|c|c|c|c|c|c|c|c|c|c|}
\hline Género y especie & CS & $\mathrm{BR}$ & $\mathrm{BC}$ & PE & EC & $\mathrm{CO}$ & VE & GU & 1 & 2 & 3 & 4 & 5 & 6 & 7 \\
\hline 269. Geonoma blanchetiana H.Wendl. ex Drude & & 1 & & & & & & & & & & 1 & & & \\
\hline 270. Geonoma brevispatha Barb.Rodr. & 1 & 1 & & & & & & & & & 1 & & & & \\
\hline 271. Geonoma brongniartii Mart. & & 1 & 1 & 1 & 1 & 1 & & & 1 & & & & & & \\
\hline 272. Geonoma calyptrogynoidea Burret & & & & & 1 & 1 & & & & & & & 1 & & \\
\hline 273. Geonoma camana Trail & & 1 & & 1 & 1 & 1 & & & 1 & & & & & & \\
\hline 274. Geonoma chlamydostachys Galeano & & & & & & 1 & & & & & & & 1 & & \\
\hline 275. Geonoma chococola Wess.Boer & & & & & & 1 & & & & & & & 1 & & \\
\hline 276. Geonoma concinna Burret & & & & & & 1 & & & & & & & 1 & 1 & \\
\hline 277. Geonoma cuneata H.Wendl. & & & & & 1 & 1 & 1 & & & & & & 1 & 1 & \\
\hline 278. Geonoma deversa (Poit.) Kunth & & 1 & 1 & 1 & 1 & 1 & 1 & 1 & 1 & 1 & & & 1 & 1 & \\
\hline 279. Geonoma divisa H.E.Moore & & & & & & 1 & & & & & & & 1 & & \\
\hline 280. Geonoma ecuadorensis A.J.Hend., Borchs. Balslev & & & & & 1 & & & & 1 & & & & & 1 & \\
\hline 281. Geonoma elegans Mart. & & 1 & & & & & & & & & & 1 & & & \\
\hline 282. Geonoma euspatha Burret & & 1 & 1 & 1 & 1 & 1 & 1 & 1 & 1 & 1 & & & & 1 & \\
\hline 283. Geonoma ferruginea H.Wendl. & & & & & & $?$ & & & & & & & $?$ & & \\
\hline 284. Geonoma frontinensis Burret & & & & & & 1 & & & & & & & & 1 & \\
\hline 285. Geonoma fusca Wess.Boer & & & & & & & & 1 & & & & & & 1 & \\
\hline 286. Geonoma gamiova Barb.Rodr. & & 1 & & & & & & & & & & 1 & & & \\
\hline 287. Geonoma gastoniana Glaz. ex Drude & & 1 & & & & & & & & & & 1 & & & \\
\hline 288. Geonoma hollinensis A.J.Hend., Borchs. \& Balslev & & & & & 1 & & & & & & & & & 1 & \\
\hline 289. Geonoma interrupta (Ruiz \& Pav.) Mart. & & & 1 & 1 & 1 & 1 & 1 & & 1 & 1 & & & 1 & 1 & \\
\hline 290. Geonoma irena Borchs. & & & & & 1 & & & & & & & & 1 & & \\
\hline 291. Geonoma lanata A.J.Hend., Borchs. \& Balslev & & & & & 1 & 1 & & & & & & & 1 & 1 & \\
\hline 292. Geonoma laxiflora Mart. & & 1 & 1 & 1 & 1 & 1 & & & 1 & & & & & & \\
\hline 293. Geonoma leptospadix Trail & & 1 & 1 & 1 & 1 & 1 & 1 & 1 & 1 & & & & 1 & & \\
\hline 294. Geonoma linearis Burret & & & & & 1 & 1 & & & & & & & 1 & & \\
\hline 295. Geonoma longepedunculata Burret & & & & 1 & 1 & 1 & & & 1 & & & & & & \\
\hline 296. Geonoma longevaginata H.Wendl. & & & & & & 1 & & & & & & & 1 & & \\
\hline 297. Geonoma macrostachys Mart. & & 1 & 1 & 1 & 1 & 1 & 1 & 1 & 1 & 1 & & & & & \\
\hline 298. Geonoma maxima (Poit.) Kunth & & 1 & 1 & 1 & 1 & 1 & 1 & 1 & 1 & & & & 1 & 1 & \\
\hline 299. Geonoma megalospatha Burret & & & 1 & 1 & 1 & & & & & & & & & 1 & \\
\hline 300. Geonoma multisecta (Burret) Burret & & & & & 1 & 1 & & & 1 & & & & & & \\
\hline 301. Geonoma occidentalis A.J.Hend. & & 1 & 1 & 1 & & & & & 1 & & 1 & & & & \\
\hline 302. Geonoma oldemanii Granv. & & 1 & & & & & & 1 & 1 & & & & & & \\
\hline 303. Geonoma oligoclona Trail. & & 1 & & & & 1 & 1 & & 1 & & & & & & \\
\hline 304. Geonoma orbignyana Mart. & & & 1 & 1 & 1 & 1 & 1 & & & & & & & 1 & \\
\hline 305. Geonoma paradoxa Burret & & & & & 1 & 1 & & & & & & & 1 & & \\
\hline 306. Geonoma paraguanensis H.Karst. & & & & & & & 1 & & & 1 & & & & 1 & \\
\hline 307. Geonoma pauciflora Mart. & & 1 & & & & & & & & & & 1 & & & \\
\hline 308. Geonoma pinnatifrons Willd. & & & & & 1 & 1 & 1 & & 1 & 1 & & & 1 & 1 & \\
\hline 309. Geonoma platybothros Burret & & & & & & 1 & & & & 1 & & & & 1 & \\
\hline 310. Geonoma poeppigiana Mart. & & 1 & 1 & 1 & 1 & 1 & & & 1 & & & & & & \\
\hline 311. Geonoma pohliana Mart. & & 1 & & & & & & & & & & 1 & & & \\
\hline 312. Geonoma procumbens H.Wendl. ex Spruce & & & & & & 1 & & & & & & & 1 & & \\
\hline 313. Geonoma pulcherrima Burret & & & & & 1 & & & & 1 & & & & & & \\
\hline 314. Geonoma pycnostachys Mart. & & 1 & 1 & 1 & 1 & 1 & 1 & 1 & 1 & & & & 1 & 1 & \\
\hline 315. Geonoma ramosissima Burret & & & & & & 1 & & & & & & & 1 & & \\
\hline 316. Geonoma rivalis Kalbreyer \& Burret & & & & & & 1 & & & & & & & 1 & & \\
\hline 317. Geonoma rubescens H.Wendl. ex Drude & & 1 & & & & & & & & & & 1 & & & \\
\hline 318. Geonoma santanderensis Galeano \& R.Bernal & & & & & & 1 & & & & & & & & 1 & \\
\hline 319. Geonoma schottiana Mart. & & 1 & & & & & & & & & & 1 & & & \\
\hline 320. Geonoma simplicifrons Willd. & & & & & & & 1 & & & 1 & & & & 1 & \\
\hline 321. Geonoma skovii A.J.Hend., Borchs. \& Balslev & & & & & 1 & & & & & & & & & 1 & \\
\hline 322. Geonoma spinescens H.Wendl. & & & & & & 1 & 1 & & & 1 & & & & 1 & \\
\hline 323. Geonoma stricta (Poit.) Kunth & & & & & & & & 1 & 1 & & & & & & \\
\hline
\end{tabular}

(continúa...) 
Apéndice 1. (continuación)

\begin{tabular}{|c|c|c|c|c|c|c|c|c|c|c|c|c|c|c|c|}
\hline Género y especie & CS & $\mathrm{BR}$ & $\mathrm{BC}$ & $\mathrm{PE}$ & EC & $\mathrm{CO}$ & VE & GU & 1 & 2 & 3 & 4 & 5 & 6 & 7 \\
\hline 324. Geonoma supracostata Svenning & & & & 1 & 1 & & & & 1 & & & & & & \\
\hline 325. Geonoma tenuissima H.E.Moore & & & & & 1 & & & & & & & & 1 & & \\
\hline 326. Geonoma triandra (Burret) Wess.Boer & & & & & 1 & 1 & & & & & & & 1 & & \\
\hline 327. Geonoma triglochin Burret & & & 1 & 1 & 1 & 1 & & & 1 & & & & & 1 & \\
\hline 328. Geonoma trigona (Ruiz \& Pav.) A.H.Gentry & & & & 1 & & & & & & & & & & 1 & \\
\hline 329. Geonoma umbraculiformis Wess.Boer & & 1 & & & & & & 1 & 1 & & & & & & \\
\hline 330. Geonoma undata Klotzsch & & 1 & 1 & 1 & 1 & 1 & 1 & 1 & & 1 & & & 1 & 1 & \\
\hline 331. Geonoma vaga Griseb. \& H.Wendl. & & & & & & & 1 & & & 1 & & & & & \\
\hline 332. Geonoma wilsonii Galeano \& R.Bernal & & & & & & 1 & & & & & & & & 1 & \\
\hline 333. Hyospathe elegans Mart. & & 1 & 1 & 1 & 1 & 1 & 1 & 1 & 1 & 1 & & & 1 & 1 & \\
\hline 334. Hyospathe frontinensis A.J.Hend. & & & & & & 1 & & & & & & & & 1 & \\
\hline 335. Hyospathe macrorhachis Burret & & & & 1 & 1 & & & & & & & & & 1 & \\
\hline 336. Hyospathe peruviana A.J.Hend. & & & & 1 & & & & & & & & & & 1 & \\
\hline 337. Hyospathe pittieri Burret & & & & & & 1 & 1 & & & & & & & 1 & \\
\hline 338. Hyospathe wendlandiana Dammer ex Burret & & & & & & 1 & & & & & & & & 1 & \\
\hline 339. Iriartea deltoidea Ruiz \& Pav. & & 1 & 1 & 1 & 1 & 1 & 1 & & 1 & 1 & & & 1 & 1 & \\
\hline 340. Iriartella setigera (Mart.) H.Wendl. & & 1 & & & & 1 & 1 & 1 & 1 & & & & & & \\
\hline 341. Iriartella stenocarpa Burret & & 1 & 1 & 1 & & 1 & & & 1 & & & & & & \\
\hline 342. Itaya amicorum H.E.Moore & & 1 & & 1 & & 1 & & & 1 & & & & & & \\
\hline 343. Jubaea chilensis (Molina) Baillon & & & 1 & & & & & & & & & & 1 & & \\
\hline 344. Leopoldinia major Wallace & & 1 & & & & 1 & 1 & & 1 & & & & & & \\
\hline 345. Leopoldinia piassaba Wallace & & 1 & & & & 1 & 1 & & 1 & & & & & & \\
\hline 346. Leopoldinia pulchra Mart. & & 1 & & & & 1 & 1 & & 1 & & & & & & \\
\hline 347. Lepidocaryum tenue Mart. & & 1 & & 1 & & 1 & 1 & 1 & 1 & & & & & & \\
\hline 348. Lytocaryum hoehnei (Burret) Tol. & & 1 & & & & & & & & & & 1 & & & \\
\hline 349. Lytocaryum weddellianum (H.Wendl.) Tol. & & 1 & & & & & & & & & & 1 & & & \\
\hline 350. Manicaria saccifera Gaertn. & & 1 & & 1 & 1 & 1 & 1 & 1 & 1 & 1 & & & 1 & & \\
\hline 351. Mauritia carana Wallace & & 1 & & 1 & & 1 & 1 & & 1 & & & & & & \\
\hline 352. Mauritia flexuosa L.f. & & 1 & 1 & 1 & 1 & 1 & 1 & 1 & 1 & 1 & 1 & & & & \\
\hline 353. Mauritiella aculeata (Kunth) Burret & & 1 & & 1 & & 1 & 1 & & 1 & & & & & & \\
\hline 354. Mauritiella armata (Mart.) Burret & & 1 & 1 & 1 & 1 & 1 & 1 & 1 & 1 & 1 & 1 & & & & \\
\hline 355. Mauritiella macroclada (Burret) Burret & & & & & & 1 & & & & & & & 1 & & \\
\hline 356. Oenocarpus bacaba Mart. & & 1 & & & & 1 & 1 & 1 & 1 & & & & & & \\
\hline 357. Oenocarpus balickii F.Kahn & & 1 & 1 & 1 & & 1 & 1 & & 1 & & & & & & \\
\hline 358. Oenocarpus bataua Mart. & & 1 & 1 & 1 & 1 & 1 & 1 & 1 & 1 & 1 & & & 1 & 1 & \\
\hline 359. Oenocarpus circumtextus Mart. & & & & & & 1 & & & 1 & & & & & & \\
\hline
\end{tabular}

360. Oenocarpus distichus Mart.

361. Oenocarpus makeru R.Bernal, Galeano \& A.J.Hend.

362. Oenocarpus mapora H.Karst.

363. Oenocarpus minor Mart.

364. Oenocarpus simplex R.Bernal, Galeano \& A.J.Hend.

365. Parajubaea cocoides Burret

366. Parajubaea sunkha M.Moraes

367. Parajubaea torallyi (Mart.) Burret

368. Pholidostachys dactyloides H.E.Moore

369. Pholidostachys kalbreyeri H.Wendl. ex Burret

370. Pholidostachys pulchra H.Wendl. ex Burret

371. Pholidostachys synanthera (Mart.) H.E.Moore

372. Phytelephas aequatorialis Spruce

373. Phytelephas macrocarpa Ruiz \& Pav.

374. Phytelephas schottii H.Wendl.

375. Phytelephas tenuicaulis (Barfod) A.J.Hend.

376. Phytelephas tumacana O.F. Cook

377. Prestoea acuminata (Willd.) H.E.Moore

378. Prestoea carderi Hook.f. 
Apéndice 1. (continuación)

\begin{tabular}{|c|c|c|c|c|c|c|c|c|c|c|c|c|c|c|c|}
\hline Género y especie & CS & BR & $\mathrm{BC}$ & $\mathrm{PE}$ & EC & $\mathrm{CO}$ & VE & GU & 1 & 2 & 3 & 4 & 5 & 6 & 7 \\
\hline 379. Prestoea decurrens (H.Wendl. ex Burret) H.E.Moore & & & & & 1 & 1 & & & & & & & 1 & 1 & \\
\hline 380. Prestoea ensiformis (Ruiz \& Pav.) H.E.Moore & & & & 1 & 1 & 1 & & & 1 & & & & 1 & 1 & \\
\hline 381. Prestoea longepetiolata (Oerst.) H.E.Moore & & & & & & 1 & 1 & & & 1 & & & & & \\
\hline 382. Prestoea pubens H.E.Moore & & & & & 1 & 1 & & & & & & & 1 & 1 & \\
\hline 383. Prestoea pubigera (Griseb. \& H.Wendl.) Hook.f. & & & & & & & 1 & & & 1 & & & & 1 & \\
\hline 384. Prestoea schultzeana (Burret) H.E.Moore & & 1 & & 1 & 1 & 1 & & & 1 & & & & & & \\
\hline 385. Prestoea simplicifolia Galeano & & & & & & 1 & & & & & & & 1 & 1 & \\
\hline 386. Prestoea tenuiramosa (Dammer) H.E.Moore & & 1 & & & & & 1 & 1 & & & & & & 1 & \\
\hline 387. Raphia taedigera (Mart.) Mart. & & 1 & & & & 1 & & & & & & & & & 1 \\
\hline 388. Reinhardtia gracilis (H.Wendl.) Drude ex Dammer & & & & & & 1 & & & & & & & 1 & & \\
\hline 389. Reinhardtia koschnyana (H.Wendl.\& Dammer) Burret & & & & & & 1 & & & & & & & 1 & & \\
\hline 390. Reinhardtia simplex (H.Wendl.) Drude ex Dammer & & & & & & 1 & & & & & & & 1 & & \\
\hline 391. Roystonea oleracea (Jacq.) O.F.Cook & & & & & & 1 & 1 & & & 1 & & & & & \\
\hline 392. Sabal mauritiiformis (H.Karst.) Griseb. ex H.Wendl. & & & & & & 1 & 1 & & & 1 & & & 1 & & \\
\hline 393. Socratea exorrhiza (Mart.) H.Wendl. & & 1 & 1 & 1 & 1 & 1 & 1 & 1 & 1 & 1 & & & 1 & & \\
\hline 394. Socratea hecatonandra (Dugand) R.Bernal & & & & & 1 & 1 & & & & & & & 1 & & \\
\hline 395. Socratea rostrata Burret & & & & 1 & 1 & 1 & & & & & & & & 1 & \\
\hline 396. Socratea salazarii H.E.Moore & & 1 & 1 & 1 & & & & & 1 & & & & & & \\
\hline 397. Syagrus botryophora (Mart.) Mart. & & 1 & & & & & & & & & & 1 & & & \\
\hline 398. Syagrus campylospatha (Barb.Rodr.) Becc. & 1 & & & & & & & & & & 1 & & & & \\
\hline 399. Syagrus cardenasii Glassman & & & 1 & & & & & & & & 1 & & & 1 & \\
\hline 400. Syagrus cearensis Noblick & & 1 & & & & & & & & & & 1 & & & \\
\hline 401. Syagrus cocoides Mart. & & 1 & & & & & & 1 & 1 & & 1 & & & & \\
\hline 402. Syagrus comosa (Mart.) Mart. & & 1 & 1 & & & & & & & & 1 & & & & \\
\hline 403. Syagrus coronata (Mart.) Becc. & & 1 & & & & & & & & & & 1 & & & 1 \\
\hline 404. Syagrus duartei Glassman & & 1 & & & & & & & & & 1 & & & & \\
\hline 405. Syagrus flexuosa (Mart.) Becc. & & 1 & & & & & & & & & 1 & & & & \\
\hline 406. Syagrus glaucescens Glaz. ex Becc. & & 1 & & & & & & & & & 1 & & & & \\
\hline 407. Syagrus graminifolia (Drude) Becc. & & 1 & & & & & & & & & 1 & & & & \\
\hline 408. Syagrus harleyi Glassman & & 1 & & & & & & & & & 1 & & & & \\
\hline 409. Syagrus inajai (Spruce) Becc. & & 1 & & & & & & 1 & 1 & & & & & & \\
\hline 410. Syagrus lilliputiana (Barb.Rodr.) Becc. & 1 & 1 & & & & & & & & & 1 & & & & \\
\hline 411. Syagrus loefgrenii Glassman & & 1 & & & & & & & & & & & & & \\
\hline 412. Syagrus macrocarpa Barb.Rodr. & & 1 & & & & & & & & & & 1 & & & \\
\hline 413. Syagrus mendanhensis Glassman & & 1 & & & & & & & & & 1 & & & & \\
\hline 414. Syagrus microphylla Burret & & 1 & & & & & & & & & 1 & & & & \\
\hline 415. Syagrus oleracea (Mart.) Becc. & 1 & 1 & & & & & & & & & & 1 & & & \\
\hline
\end{tabular}

416. Syagrus orinocensis (Spruce) Burret

417. Syagrus petraea (Mart.) Becc.

418. Syagrus picrophylla Barb.Rodr.

419. Syagrus pleioclada Burret

420. Syagrus pseudococos (Raddi) Glassman

421. Syagrus romansoffiana (Cham.) Glassman

422. Syagrus ruschiana (Bondar) Glassman

423. Syagrus sancona (Kunth) H.Karst.

424. Syagrus schizophylla (Mart.) Glassman

425. Syagrus smithii (H.E. Moore) Glassman

426. Syagrus stenopetala Burret

427. Syagrus stratincola Wess.Boer

428. Syagrus vagans (Bondar) A.D.Hawkes

429. Syagrus vermicularis Noblick

430. Syagrus werdermannii Burret

431. Syagrus yungasensis M.Moraes

432. Synechanthus warscewiczianus H.Wendl.

$\begin{array}{lll}1 & 1 & 1\end{array}$

1

11

11

1

1

1

1

1

1

1

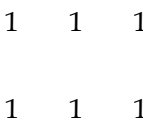

1

1

1

11

1

1 $\begin{array}{ll}1 & \\ & 1\end{array}$

1

1

$1 \quad 1$

1


Apéndice 1. (continuación)

\begin{tabular}{|c|c|c|c|c|c|c|c|c|c|c|c|c|c|c|c|}
\hline Género y especie & CS & $\mathrm{BR}$ & $\mathrm{BC}$ & PE & $\mathrm{EC}$ & $\mathrm{CO}$ & VE & GU & 1 & 2 & 3 & 4 & 5 & 6 & 7 \\
\hline 433. Trithrinax brasiliensis Mart. & 1 & 1 & & & & & & & & & 1 & 1 & & & \\
\hline 434. Trithrinax campestris (Burmeister) Drude \& Griseb. & 1 & & & & & & & & & & 1 & & & & \\
\hline 435. Trithrinax schizophylla Drude & 1 & 1 & 1 & & & & & & & & 1 & & & 1 & \\
\hline 436. Welfia regia H.Wendl. ex André & & & & $?$ & 1 & 1 & & & $?$ & & & & 1 & 1 & \\
\hline 437. Wendlandiella gracilis Dammer & & 1 & 1 & 1 & & & & & 1 & & & & & & \\
\hline 438. Wettinia aequalis (O.F.Cook \& Doyle) R. Bernal & & & & & 1 & 1 & & & & & & & 1 & & \\
\hline 439. Wettinia aequatorialis R.Bernal & & & & $?$ & 1 & & & & & & & & & 1 & \\
\hline 440. Wettinia anomala (Burret) R.Bernal & & & & & 1 & 1 & & & & & & & & 1 & \\
\hline 441. Wettinia augusta Poepp. \& Endl. & & 1 & 1 & 1 & & 1 & & & 1 & & & & & 1 & \\
\hline 442. Wettinia castanea H.E.Moore \& J.Dransf. & & & & & & 1 & & & & & & & & 1 & \\
\hline 443. Wettinia disticha (R.Bernal) R.Bernal & & & & & & 1 & & & & & & & & 1 & \\
\hline 444. Wettinia drudei (O.F.Cook \& Doyle) A.J.Hend. & & 1 & & 1 & 1 & 1 & & & 1 & & & & & & \\
\hline 445. Wettinia fascicularis (Burret) H.E.Moore \& J.Dransf. & & & & & 1 & 1 & & & & & & & & 1 & \\
\hline 446. Wettinia hirsuta Burret & & & & & & 1 & & & & & & & 1 & 1 & \\
\hline 447. Wettinia kalbreyeri (Burret) R.Bernal & & & & & 1 & 1 & & & & & & & 1 & 1 & \\
\hline 448. Wettinia lanata R.Bernal & & & & & & 1 & & & & & & & & 1 & \\
\hline 449. Wettinia longipetala A.H.Gentry & & & & 1 & 1 & & & & 1 & & & & & 1 & \\
\hline 450. Wettinia maynensis Spruce & & & & 1 & 1 & 1 & & & 1 & & & & & 1 & \\
\hline 451. Wettinia microcarpa (Burret) R.Bernal & & & & & & 1 & & & & & & & & 1 & \\
\hline 452. Wettinia minima R.Bernal & & & & 1 & 1 & & & & & & & & & 1 & \\
\hline 453. Wettinia oxycarpa Galeano \& R.Bernal & & & & & 1 & 1 & & & & & & & 1 & 1 & \\
\hline 454. Wettinia praemorsa (Willd.) Wess.Boer & & & & & & 1 & 1 & & & 1 & & & & 1 & \\
\hline 455. Wettinia quinaria (O.F.Cook \& Doyle) Burret & & & & & 1 & 1 & & & & & & & 1 & & \\
\hline 456. Wettinia radiata (O.F. Cook \& Doyle) R.Bernal & & & & & 1 & 1 & & & & & & & 1 & & \\
\hline 457. Wettinia verruculosa H.E.Moore & & & & & 1 & 1 & & & & & & & & 1 & \\
\hline Género y especie & CS & $\mathrm{BR}$ & $\mathrm{BC}$ & $\mathrm{PE}$ & $\mathrm{EC}$ & $\mathrm{CO}$ & VE & GU & 1 & 2 & 3 & 4 & 5 & 6 & 7 \\
\hline
\end{tabular}




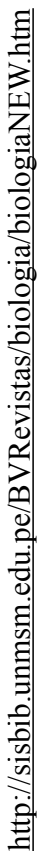

